\title{
Pricing when customers have limited attention
}

Tamer Boyacı, ESMT European School of Management and Technology

Yalçın Akçay, College of Administrative Sciences and Economics, Koç University

Revised version

Copyright 2016 by ESMT European School of Management and Technology GmbH, Berlin, Germany, www.esmt.org. All rights reserved. No part of this publication may be reproduced, stored in a retrieval system, used in a spreadsheet, or transmitted in any form or by any means - electronic, mechanical, photocopying, recording, or otherwise - without the permission of ESMT.

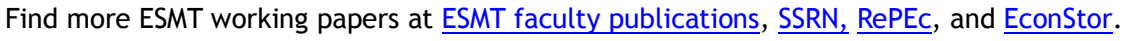




\title{
Submitted to Management Science \\ manuscript MS-15-00653.R3
}

\section{Pricing When Customers Have Limited Attention}

\author{
Tamer Boyac1 \\ ESMT European School of Management and Technology, \\ Schlossplatz 1, 10178, Berlin, Germany \\ Phone: +49 30 21231-1294; Fax: +49 30 21231-1281 \\ tamer.boyaci@esmt.org \\ Yalçın Akçay \\ College of Administrative Sciences and Economics, Koç University, \\ Rumeli Feneri Yolu, Istanbul, 34450, Turkey, \\ Phone: (90) 212338 1560; Fax: (90) 2123381393 \\ yakcay@ku.edu.tr
}

\begin{abstract}
We study the optimal pricing problem of a monopolistic firm facing customers with limited attention and capability to process information about the value (quality) of a single offered product. We model customer choice based on the theory of rational inattention in the economics literature, which enables us to capture not only the impact of true quality and price, but also the intricate effects of customer's prior beliefs and cost of information acquisition and processing. We formulate the firm's price optimization problem assuming that the firm can also use the price to signal the quality of the product to customers. To delineate the economic incentives of the firm, we first characterize the pricing and revenue implications of customer's limited attention without signalling, and then use these results to explore Perfect Bayesian Equilbiria (PBE) of the strategic pricing signalling game. As an extension, we consider heterogeneous customers with different information costs as well as prior beliefs. We discuss the managerial implications of our key findings and prescribe insights regarding information provision and product positioning.
\end{abstract}

Key words: pricing, choice behaviour, rational inattention, information acquisition, signalling game

\section{Introduction}

We are living in an era characterized by abundance of information. Firms are in a race to make use of the "big data" available on their customers and products. In a similar vein, customers have many products/services to choose from and have easy access to a wealth of information sources that can aid in their decision processes. In theory, all the information that is available on the internet, social 
media, product catalogs, magazines and other published media, information broadcasted on radio, television, information obtained from friends and family are at the disposal of customers. However, almost by default, customers not only have limited time and attention, but also limited capability to process the information that is acquired. Therefore, information acquisition and processing is a "costly" endeavor. Consequently, customers have to choose how much and what type of information to pay attention to (and what to ignore), and make purchase decisions on the basis of this partial information. Understanding such limitations and how they translate into choice behavior is of crucial concern to the selling firm (e.g., a retailer) since there are immediate implications on the pricing and information provision strategies. These constitute the primary focus of our paper.

For exemplary purposes, consider a customer who is in the market to buy a digital camera. It is relatively easy to obtain information regarding the selection of brands, models and associated prices from an on-line retailer or by walking into a physical store. Yet, cameras differ significantly in terms of features (resolution, optical/digital zoom, battery life, etc.) and performance (color reproduction, noise, distortion, etc). The customer might have some past experience with the brands and some knowledge about product features, which form her prior belief about the "quality" levels of these cameras. She might even attempt to infer product quality by carefully interpreting prices. Naturally the customer can also learn more about the products by asking specific questions to the seller, searching facts and comparisons on-line, etc. However, she has limited time and capacity to devote to this task, hence each attempt to acquire additional information represents a cost to the customer. The customer has to determine how much effort to spend to acquire quality revealing information, what information to pay attention to and process while making the final decision. For the seller, the key question is how to set prices when faced with such customers.

In order to examine the optimal pricing strategies of the seller, it is essential to capture the salient features of limited attention and information processing capabilities of customers in a choice model. Rational inattention theory (Sims 1998, 2003, 2006) offers a compelling approach for this purpose. In contrast to the rational expectations theory which assumes that customers can fully process all freely 
Author: Pricing When Customers Have Limited Attention

available information about the product, rational inattention theory assumes that they lack the capability to understand the available information comprehensively and translate it into decisions. ${ }^{1}$ At the core of rational inattention is the understanding that attention is a scarce resource and therefore must be allocated wisely. In particular, the pioneering works of Sims (1998, 2003, 2006) propose a framework that is based on a stream of literature on information theory, which measures uncertainty by entropy and quantifies information as reduction in uncertainty. This approach does not make particular assumptions on how decision-makers acquire information and what they learn. It builds on utility-maximizing decision-makers who acquire information optimally, trading off the expected benefit of better information against the cost associated with acquiring it. Accordingly, rationally inattentive decision-makers optimally select the type and quantity of information they need, and ignore the information that is not worth obtaining and processing. ${ }^{2}$ In fact, in a recent paper, Matějka and McKay (2015) show that when faced with discrete choices with stochastic (pay-off) values, a rationally inattentive decision-maker's optimal information processing strategy endogenously leads to a choice behavior that can be characterized as generalized multinomial logit (MNL). In particular, the choice probabilities depend not only on the true realizations of the choices, but also on the prior belief of the customer and the cost of information. We utilize this characterization of the optimal choice in our pricing framework.

We remark that rational inattention theory has been applied to a broad spectrum of economic problems and has been a powerful construct in providing explanations to some observed market and macroeconomic phenomena that extant theories fail to fully address, such as business cycles (McKowiak and Wiederholt 2015), consumption (Tutino 2013), price setting behavior and stickiness (Matějka 2015). The main obstacle preventing much wider applications has been the mathematical and computational complexity associated with models utilizing the rational inattention framework (Tutino 2011). As such, optimal firm-level pricing decisions in the presence of customers with limited attention, and how such behaviour impacts prices and profitability are widely unknown. We aim to bridge this gap. To this end, we seek answers to the following research questions:

\footnotetext{
${ }^{1}$ Throughout the paper we use the terms "limited attention" and "rational inattention" interchangeably.

${ }^{2}$ We discuss this theory in the context of our problem in more detail in $\S 3$.
} 
- How should a firm price its products when customers have limited attention and cannot fully evaluate the quality of the products it offers?

- What are the implications of the firm "signalling" product quality to inattentive customers through its product pricing strategy?

- What is the impact of key market and operational factors, such as customers' prior beliefs about product quality, cost of information, on the firm's revenue and the products' prices?

- What should or could the firm do in terms of its product positioning and information provision efforts to enhance its revenue when faced with rationally inattentive customers?

In order to address these questions, we develop a model with a single firm selling a single product to a homogenous population of rationally inattentive customers. Customers can observe the price of the product, but are not completely aware of and cannot fully assess its quality. In line with rational inattention literature discussed above, customers in our model have prior beliefs about the quality level of the product and can improve their assessment by optimally acquiring and processing costly information (which is captured in our particular discrete choice model). The firm, on the other hand, has more experience with the product and knows its exact quality. It can also strategically signal the quality of the product to its customers through the price and influence their prior beliefs about quality in the process. We formulate the problem as a sequential-move pricing signalling game, in which the respective strategies of the firm and the customers are defined as their pricing and purchasing decisions, respectively. We first comprehensively analyze the firm's price optimization problem without the signalling component, and fathom the key drivers of the firm's economic incentives when customers have limited attention. Building on these insights, we then explore the Perfect Bayesian Equilibria (PBE) that can arise when prices signal quality. Moreover, we incorporate customer heterogeneity into our model by allowing a mix of attentive and inattentive customers in the market, and by allowing customers to have different prior beliefs.

Our contribution to the literature is three-fold: (i) From a theoretical perspective, utilizing recent advances in the theory of rational inattention, we develop a tractable price optimization framework that captures the limited attention of customers. (ii) Utilizing our model, we characterize the pricing 
Author: Pricing When Customers Have Limited Attention

Article submitted to Management Science; manuscript no. MS-15-00653.R3

and revenue implications of customer's limited attention in the presence of prices signalling quality, describe how key problem features (cost of information, prior beliefs, product quality) shape these decisions, and test the robustness of our results under heterogenous customers. (iii) Our descriptive results have prescriptive value since they offer valuable insights to practicing managers. The impact of customer's cost of information can be translated into suitable information provision strategies for the firm. Hence, we can identify settings in which it is profitable for the firm to make it easier for customers to gather information about products (e.g., via salesforce, trial samples, etc) and vice versa. Likewise, we impart insights that can guide product positioning efforts of the firm.

\section{Literature Review}

It has been long recognized that rationality of decision-makers is limited by the partial information they have, their cognitive capacities, and the finite amount of time they have to make decisions. Simon (1955) introduced "bounded rationality" as an alternative to modeling rationality as optimization, which views decision making as a fully rational process of finding an optimal choice given the information available. He proposed that decision-makers apply rationality after simplifying their available choices, and only seek a satisfactory choice rather than the optimal one (satisficing rather than optimizing). Since then, the field of behavioral economics has expanded significantly and has found applications in other disciplines, including operations management. Özer and Zheng (2012) provides a comprehensive coverage of behavioral models related to pricing and price contracting among firms.

Our paper is more closely related to the growing body of economics literature that explains the limited rationality of decision-makers as an implication of their limited capacity to process information (Shannon 1948). "Rational inattention" theory, as proposed by Sims (1998, 2003, 2006), rationalizes the choice behaviour of decision-makers who choose their preferred amount of information and allocation of attention, when all options and information pertaining to them are fully available, but attending to and processing this information is costly (i.e. information frictions). As noted in Wiederholt (2010), rational inattention has been applied to numerous areas in macroeconomics. Within this body of work, the more relevant for our paper is the stream that applies it to price setting. Most papers in this stream have a monetary policy focus, consider sellers who are rationally inattentive to prices and show 
how optimal prices in the market may behave "sticky" or "rigid" (Mackowiak and Wiederholt 2009, Woodford 2009, Matějka 2016). Matějka (2015) extends the notion of price rigidity to the case where customers are inattentive to prices.

In the realm of pricing under rational inattention, Matějka and McKay (2012) is akin to our work since it is also based on the generalized MNL characterization of rationally inattentive customers' response to discrete choices in Matějka and McKay (2015). Specifically, it considers multiple sellers that face random input costs and produce random quality products. Based on the realized cost and quality levels, sellers set prices (competitively), but customers are rationally inattentive to prices. Given the complexity of the general problem, the analysis is concentrated on very special cases, and the discussion centers around the role of prior knowledge and heterogeneity. In contrast, in our paper customers are able to perceive product prices perfectly, but are rationally inattentive to quality, i.e., different features and performance dimensions of the product. We are able to explicitly solve the rationally inattentive customers' decision problem, and formulate and analyze comprehensively the seller's revenue maximization problem under different settings.

By the very nature of the problem we study, our paper is also related to two vast streams of literature: (i) information search and (ii) quality uncertainty. Costly information search has been a rich area of investigation going back as early as Stigler (1961), with different approaches to modeling the acquisition and processing of information. On one hand, in fixed sample size search models, consumers first determine a sample of products (akin to a consideration set in consumer behavior) about which to gather information and then make a purchase decision (Roberts and Lattin 1991, Manzini and Mariotti 2014, Sahin and Wang 2014). On the other hand, in sequential search models, customers learn the value of alternatives (or gather further information about a certain product) one-by-one, and make a purchase after deciding optimally to stop collecting more information (Weitzman 1979, Branco et al. 2012). Our paper is in spirit similar to a sequential search, however it follows the rational inattention modelling approach to information frictions. It is also distinct in that it combines product quality uncertainty and costly information acquisition induced by limited attention in the study of optimal pricing. Starting with the seminal work of Akerlof (1970), many papers considering quality uncertainty 
Author: Pricing When Customers Have Limited Attention

focus on the information asymmetry between customer and the firm. In particular, they show how this asymmetry impacts market structure (Bester 1998) and explore how the firm signals quality via different channels such as prices (Bagwell and Riordan 1991), price endings (Stiving 2000), advertising (Erdem et al. 2008), scarcity (Stock and Balachander 2005), and queue lengths (Debo et al. 2012, Debo and Veeraraghavan 2014). In this stream Bester and Ritzberger (2001) is particularly relevant since it combines information search with quality uncertainty. Specifically, it allows the customers to pay a search cost and fully reveal the quality of the product, in a price signalling context. There are some recent efforts to incorporate rational inattention in this context. Roesler (2015) investigates the impact of customer's information strategy on optimal prices in a model where customers commit to their information acquisition in advance. Martin (2016) on the other hand utilizes an alternative approach (posterior-based) to model inattention and imposes a price cap on the prices that can be charged. Focusing on the semi-pooling/separating equilibria, the paper investigates the informativeness of the equilibrium prices as a function of information costs. In contrast, we utilize the optimal consumer choice under rational inattention (GMNL) with no price restrictions and maintain focus on the optimal pricing strategies and revenue implications of inattention. We do so by analyzing pure strategies, different mixed equilibrium strategies, as well as the case without signalling.

\section{Model Formulation and Analysis}

Consider a monopolistic firm selling a single product to rationally inattentive customers. For expositional clarity, cost of production is taken as zero, and the total market size is normalized to 1. Customers observe the price $p$ of the product, but they are not fully aware of the true quality of the product denoted as $q$. The firm, on the other hand, has more experience with the product and knows the true quality nature of the product. For analytical tractability, we assume a simple canonical form with two quality levels: high $\left(q=q_{H}\right)$ and low $\left(q=q_{L}\right)$, where $q_{H}>q_{L} \geq 0$. The customer's prior belief, denoted by distribution $G$, is such that the product is high-quality $\left(q=q_{H}\right)$ with probability $\alpha(0<\alpha<1)$, and low-quality $\left(q=q_{L}\right)$ with probability $(1-\alpha)$. These assumptions enable us to focus conspicuously on the interesting case where customers face ex-ante some uncertainty about product quality. We denote the net value of a product with quality $q_{i}, i \in\{H, L\}$, and price $p$ as $v=q_{i}-p$ (Tirole 1988), and 
normalize the net value of the outside option to zero $(v=0$ when the customer does not purchase the product). This implies that firm will only consider prices $p \in\left[q_{L}, q_{H}\right]$. Customers can acquire and process information about the quality level at a unit cost $\lambda>0$ (we assume that customers are homogenous in their information processing costs and prior beliefs, and defer the discussion of the issues regarding customer "heterogeneity" to $§ 4)$. Meanwhile, the firm can also use the price to strategically "signal" the quality of the product to the inattentive customers. As a result, we search for Perfect Bayesian Equilibria (PBE) of the following strategic pricing signalling game: The firm first decides on the product price which is freely observed by the customers. After observing the price of the product, customers revise their prior beliefs, establishing interim beliefs about the quality of the product. Then, they optimally acquire and process information about the quality, and make their optimal purchase decisions accordingly. Firm profit is realized based on the optimal purchasing behavior of the customers.

In this sequential-move game, the firm's strategy is the pricing decision $p, p \in\left[q_{L}, q_{H}\right]$. Let us define the probability that a firm with a high-quality product (also referred to as a high-quality firm) charges price $p$ as $\sigma_{H}(p)$. Similarly, let $\sigma_{L}(p)$ indicate the probability that a firm selling a low-quality product (also referred to as a low-quality firm) sets the price as $p$. The customer's strategy, on the other hand, is the purchasing decision, i.e., the probability that a customer buys the product when the price charged is $p$. This probability naturally depends on the true quality of the product. The customer's decision process is as follows. After observing price $p$, the customer updates her prior belief and forms an interim belief about the quality of the product. Specifically, let $\mu(p)$ denote the probability that the customers believe that the observed price $p$ is associated with a high-quality product, and $G^{\mu}$ denote the distribution of the interim belief.

We define $\pi_{i}^{\mu}(p)$ as the customer's optimal purchase probability of the product at price $p$ when her interim belief is $\mu(p)$, and the true quality level is $q_{i}, i \in\{H, L\}$. Note that if $\mu(p) \in\{0,1\}$, the customer essentially makes the purchase decision under perfect information (deterministic product quality), i.e., $\pi_{i}^{\mu}(p)=1$ if $p \leq q_{i}$, and $\pi_{i}^{\mu}(p)=0$ otherwise. On the other hand, if $\mu(p) \in(0,1)$, the customer has to trade-off the value of information agains its cost, in line with the theory of rational inattention. Accordingly, let $\mathcal{R}_{H}(p, \mu(p))=p \times \pi_{H}^{\mu}(p)$ and $\mathcal{R}_{L}(p, \mu(p))=p \times \pi_{L}^{\mu}(p)$ denote the expected profits for 
the high- and low-quality firms as functions of the price $p$ and the interim belief $\mu(p)$, respectively. We represent the PBE of the game by $\{\hat{\sigma}, \hat{\pi}, \hat{\mu}\}$, where $\hat{\sigma}_{i}(p), i \in\{H, L\}$, denotes the probability that a firm selling a product with quality level $q_{i}, i \in\{H, L\}$, sets the price as $p$ in equilibrium, $\hat{\pi}_{i}(p), i \in\{H, L\}$, denotes the equilibrium purchase probability of a product with quality $q_{i}, i \in\{H, L\}$, at price $p$, and $\hat{\mu}(p)$ denotes the customers' interim belief in equilibrium at price $p$. We characterize $\{\hat{\sigma}, \hat{\pi}, \hat{\mu}\}$ as follows:

1. The firm assigns positive probability only to prices that maximize its profit:

$$
\begin{gathered}
\mathcal{R}_{H}(p, \hat{\mu}(p)) \geq \mathcal{R}_{H}\left(p^{\prime}, \hat{\mu}\left(p^{\prime}\right)\right) \forall p^{\prime} \in\left[q_{L}, q_{H}\right] \text { whenever } \hat{\sigma}_{H}(p)>0 \\
\mathcal{R}_{L}(p, \hat{\mu}(p)) \geq \mathcal{R}_{L}\left(p^{\prime}, \hat{\mu}\left(p^{\prime}\right)\right) \forall p^{\prime} \in\left[q_{L}, q_{H}\right] \text { whenever } \hat{\sigma}_{L}(p)>0
\end{gathered}
$$

2. Customers make purchase decisions optimally:

$$
\begin{gathered}
\hat{\pi}_{H}(p)=\pi_{H}^{\hat{\mu}}(p) \\
\hat{\pi}_{L}(p)=\pi_{L}^{\hat{\mu}}(p)
\end{gathered}
$$

3. Customer beliefs are consistent with the Bayes' rule whenever possible:

$$
\hat{\mu}(p)=\frac{\alpha \hat{\sigma}_{H}(p)}{\alpha \hat{\sigma}_{H}(p)+(1-\alpha) \hat{\sigma}_{L}(p)} \text { whenever } \hat{\sigma}_{H}(p)+\hat{\sigma}_{L}(p)>0 .
$$

In the next section, §3.1, we present a synopsis of the application of rational inattention to discrete choice in our particular context. Based on the resulting choice model, in $\S 3.2$, we study the firm's pricing decision without the signalling component of the problem, i.e., $\mu(p)=\alpha$ and $G^{\mu}=G$. Using these results, we then explore, in $§ 3.3$, the PBE pertaining to the signalling game between the firm and customers with limited inattention.

\subsection{Purchase Decision Under Limited Attention}

Consider a customer facing two distinct choice options (whether to purchase the product or not). She has observed the price $p$ and formed the interim belief that the product is of high quality as $\mu(p)$. Consider the case where $\mu(p) \in(0,1)$. Since the customer has imperfect knowledge of the true quality of the product, her payoff from the purchase decision is uncertain. She can further inquire about the true state of the nature $v$ and can receive a signal $s$ to update her belief (in a Bayesian manner), and choose the option that maximizes her expected payoff. For any posterior distribution $B$, let $V(B)$ denote the expected payoff from choosing the best option. 
The novelty of the rational inattention approach is that the customer is allowed to choose the information processing strategy prior to observing the signal (i.e., information structure is completely endogenous). The information strategy of the customer is the selection of the joint distribution $F(s, v)$ of the signal and state. The customer determines the distribution $F(\cdot, \cdot)$ optimally, maximizing her ex-ante expected payoff $V(F)$ minus the cost of information $\mathcal{C}(F)$ associated with generating signals of different precision levels. The only requirement is that the marginal distribution over the states equals the distribution $G^{\mu}$, so that the customer's posterior beliefs are consistent with her (interim) beliefs.

Following the works of Sims (1998, 2003, 2006), the rational inattention literature assumes that customers process information through channels with limited capacity. Accordingly, the cost of information is given by $\mathcal{C}(F)=\lambda \kappa$, where $\lambda$ is the unit cost of acquiring and processing information that the customer deems useful (simply referred to as cost of information hereon), and $\kappa$ is the amount of information processed. Information acquisition is quantified in terms of reduction in uncertainty, which is measured by (Shannon) entropy. ${ }^{3}$ In our context, the customer is sharpening her belief on the realized value of $v$ by acquiring and processing information. The amount of information processed $\kappa$ is then the expected difference between the entropy of interim belief and the posterior entropy after observing the signal. This difference is referred to as the (Shannon) mutual information between the actual state and the signal. Mathematically speaking, letting $H(\cdot)$ denote the entropy, we have

$$
\mathcal{C}(F)=\lambda\left(H\left(G^{\mu}\right)-E_{s}[H(F(\cdot \mid s))]\right) .
$$

According to the above specification, the customer is optimally choosing: (i) how much attention to pay, i.e., how much information to process; (ii) what to pay attention to, i.e. what information to process; and (iii) what action to select given the information. As Matějka and McKay (2015) argues, this process is akin to asking a series of "yes-or-no questions" with a cost associated with each question. The more questions the customer asks, the tighter her posterior beliefs become. ${ }^{4}$ Furthermore,

\footnotetext{
${ }^{3}$ For any distribution $X$ with pdf $f$, entropy is defined as $-\int_{x} f(x) \ln (f(x)) d x$.

${ }^{4}$ From the coding theorem of information theory (Shannon 1948), the cost function (6) is proportional to the expected number of questions needed to implement the information strategy.
} 
Author: Pricing When Customers Have Limited Attention

it is shown that an optimal information strategy would generate only one posterior belief for each alternative, which allows the consumer's problem to be reformulated in terms of conditional choice probabilities directly. Solving this problem, Matějka and McKay (2015) establish in Theorem 1 that for any information cost $\lambda>0$, the optimal information processing strategy of the customer results in conditional choice probabilities that satisfy a generalized multinomial logit (GMNL) formula. Applying this characterization to our single product case, the conditional probability $\pi_{i}^{\mu}(p)$ that the customer purchases the product at price $p$, given that true quality level of the product is $q_{i}, i \in\{H, L\}$ can be stated as

$$
(\mathbf{G M N L}) \quad \pi_{i}^{\mu}(p)=\frac{\pi_{0}^{\mu}(p) e^{\left(q_{i}-p\right) / \lambda}}{\pi_{0}^{\mu}(p) e^{\left(q_{i}-p\right) / \lambda}+\left(1-\pi_{0}^{\mu}(p)\right)} \quad \text { almost surely }
$$

When $\lambda=0$, the customer purchases the product with probability 1 if $p<q_{i}$. Here, $\pi_{0}^{\mu}(p)$ is the unconditional choice probability, which is given as

$$
\pi_{0}^{\mu}(p)=\mu(p) \pi_{H}^{\mu}(p)+(1-\mu(p)) \pi_{L}^{\mu}(p)
$$

We remark that the GMNL characterization in (7) does not provide a complete explicit solution to the choice probability. This is because $\pi_{0}^{\mu}(p)$ is not exogenous, but is part of the of the customer's decision making process. One needs to solve (7) together with (8) to determine the exact conditional choice probability. Note that the unconditional choice probability, $\pi_{0}^{\mu}(p)$, characterizes the choice behaviour prior to processing any information. It is by definition independent of the state of the nature, but does depend on the interim belief $G^{\mu}$ and cost of information $\lambda$.

The GMNL formula (7) captures the intricate effects of three factors under endogenous information acquisition and processing: beliefs, payoffs associated with the options, and cost of information. It is possible that an option with a low (high) true payoff is chosen with high (low) likelihood, if the customer's (interim) belief is quite favorable (unfavorable). Indeed, it can be shown that $\pi_{i}^{\mu}(p), i \in$ $\{H, L\}$, is increasing in $\mu(p)$. In addition, as the cost of information increases, the customer learns less about the true nature and relies more on her beliefs. As such, the conditional purchase probabilities might increase, decrease, or can even be non-monotone in $\lambda$. 
We next present a reduction of the GMNL framework to two commonly used choice models: standard MNL and optimal sequential search. As shown in Matějka and McKay (2015), when the customer is indifferent to different options in her interim belief, she forms unconditional choice probabilities that are uniform (i.e., $\pi_{0}^{\mu}(p)=\frac{1}{2}$ in our case), and the GMNL reduces to the standard MNL. This connection is particularly relevant in our context because a rather common approach to model bounded rationality of customers (e.g., Su 2008, Chen et al. 2012) is to adopt the quantal choice model of Luce (1959) (see McKelvey and Palfrey 1995). Despite this connection, the choice behaviour under the two models can be quite different. This is mainly because rational inattention is micro-founded and the properties of probabilistic choice change when the environment changes (e.g., prior beliefs change, information becomes harder to obtain). In contrast, the quantal choice motivated MNL offers a macro-view of the choice process under information frictions, capturing the notion that customers choose better options with higher probability. Another major distinction can be observed when some options are a-priori very similar, and in particular identical. The GMNL choice behavior of the rationally inattentive customer does not follow the Independence of Irrelevant Alternatives (IIA) Axiom of Luce (1959) which generates the MNL model (see Matějka and McKay 2015). A rationally inattentive customer ignores duplicate options and treats them jointly as one option. ${ }^{5}$ There are other appealing features of the choice behaviour under rational inattention that sets it apart. For example, adding an option to the choice set can increase the likelihood that an existing choice is selected, which is not possible under MNL or other random utility choice models (Matějka and McKay 2015). Likewise, GMNL model is shown to be consistent with a standard theory of imperfect perception based on signal processing and choice. Hence it can be rationalized within a standard Bayesian context, while MNL and consideration set based models cannot be (Caplin and Martin 2014).

Consider now a relaxation of the information acquisition process of the inattentive customer, and assume that she can generate the perfect signal with complete information. In other words, the signal reveals the true state of the nature, and therefore the expected posterior entropy is $E_{s}[H(F(\cdot \mid s))]=0$.

${ }^{5}$ Different generalizations of the MNL model has been proposed in the literature to remedy the IIA problem, such as the nested logit, mixed MNL. See for example Aksoy-Pierson et al. (2013) for a discussion. 
Author: Pricing When Customers Have Limited Attention

Article submitted to Management Science; manuscript no. MS-15-00653.R3

In this case, the decision problem boils down to the choice of paying the information cost $\mathcal{C}(F)=$ $\lambda H\left(G^{\mu}(v)\right)$ and choosing the option based on true realization, or not paying the cost and making a decision on the basis of interim expectations. This particular decision making process of the customer is akin to a sequential information search. Observe that the optimal information search strategy would yield a binary choice behaviour (probability of choosing an option is either zero or one). In contrast, under rational inattention, signals are never perfect (some uncertainty always remains), and the customer displays a probabilistic choice behaviour. In this regard, the sequential search driven choice model captures the rational decision-maker's costly acquisition of information, but in doing so ignores the frictions due to limited attention and limited processing capabilities of the customers.

\subsection{Pricing without Signalling}

In this section, we study the case in which the customers do not revise their prior beliefs about product quality upon observing the price, i.e., $\mu(p)=\alpha$ and $G^{\mu}=G$. In reality, it might indeed be the case that prices are not informative of quality. Some researchers (e.g. Stafford and Enis 1969, Wheatley and Chiu 1977, Obermiller 1988) argue that consumers only use price as a signal of quality when other factors such as brand name, store reputation, product information are absent. Moreover, without signalling, we are able to delineate the economic incentives of the firm and provide insights on how underlying factors (e.g. quality levels, cost of information) are likely to impact the firm's pricing strategy. As such, these results form the building blocks of the analysis of the signalling game between the firm and the customers in $\S 3.3$.

For expositional simplicity, we drop the superscript $\mu$ from our notation in the subsequent analysis. Substituting $\pi_{H}(p)$ and $\pi_{L}(p)$ from (7) into (8), we find three possible solutions to $\pi_{0}(p)$ as

$$
\pi_{0}(p) \in\left\{0,1, \dot{\pi}_{0}(p)\right\}
$$

where

$$
\dot{\pi}_{0}(p):=\frac{e^{p / \lambda}\left(e^{p / \lambda}-(1-\alpha) e^{q_{L} / \lambda}-\alpha e^{q_{H} / \lambda}\right)}{\left(e^{p / \lambda}-e^{q_{H} / \lambda}\right)\left(e^{p / \lambda}-e^{q_{L} / \lambda}\right)} .
$$

The two solutions $\pi_{0}(p) \in\{0,1\}$ capture the cases when the customer makes the purchase decision without processing any information. If $\pi_{0}(p)=0$, the customer chooses the outside option, whereas if 
$\pi_{0}(p)=1$, the customer purchases the product. On the other hand, $\pi_{0}(p)=\dot{\pi}_{0}(p)$ describes the case when the customer processes information to make a "random" purchase decision. As shown in Matějka and McKay (2015), when there are two non-identical options (as in our case), the solution is unique.

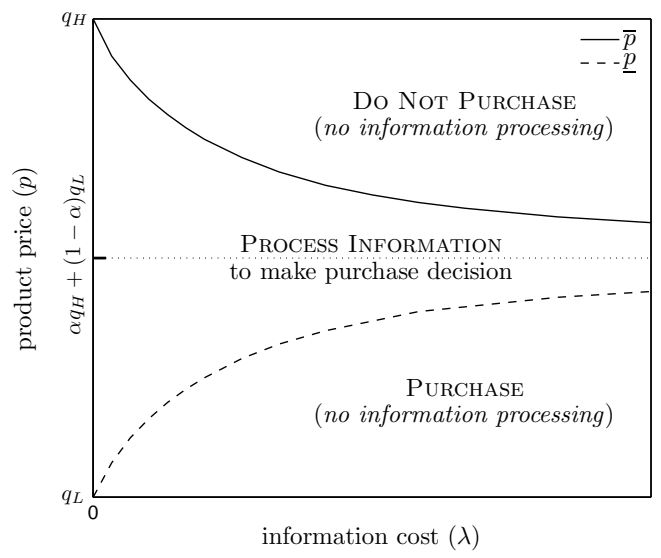

Figure 1 Product purchase and information processing strategies of an inattentive customer

Figure 1 illustrates the three possible product purchase and information processing strategies of an inattentive customer as product price and information cost varies. Clearly, if $p \leq q_{L}$ or $p \geq q_{H}$, the customer does not need to process any information (purchase if $p \leq q_{L}$, and not purchase if $p>q_{H}$ ). Note that $\dot{\pi}_{0}(p)=0$ when $p=\bar{p}:=\lambda \ln \left[\alpha e^{q_{H} / \lambda}+(1-\alpha) e^{q_{L} / \lambda}\right]$, and $\dot{\pi}_{0}(p)=1$ when $p=\underline{p}:=q_{L}+q_{H}-$ $\lambda \ln \left[(1-\alpha) e^{q_{H} / \lambda}+\alpha e^{q_{L} / \lambda}\right]$. Namely, $\dot{\pi}_{0}(\bar{p})=0$ and $\dot{\pi}_{0}(\underline{p})=1$. It can be easily verified that $\underline{p}>q_{L}$, while $\bar{p}<q_{H}$. Furthermore, $\dot{\pi}_{0}(p)>1$ if $p<\underline{p}$, and $\dot{\pi}_{0}(p)<0$ if $p>\bar{p}$. Accordingly, the customer processes information, only when $\underline{p}<p<\bar{p}$. Now, given that the customer does not process information, she purchases the product if and only if her expected utility is at least as high as the outside option, i.e. $p \leq \alpha q_{H}+(1-\alpha) q_{L}$. In Lemma 1 in Appendix, we establish that $\underline{p} \leq \alpha q_{H}+(1-\alpha) q_{L} \leq \bar{p}$. This suggests that for $q_{L}<p<\underline{p}, \pi_{0}(p)=1$ and for $\bar{p}<p<q_{H}, \pi_{0}(p)=0$, as seen in Figure 1. One can derive analogous thresholds for $\lambda, \alpha$ and $q_{H}-q_{L}$ for which customers make purchase decision without processing information. However, we omit these for brevity.

In light of above, we can explicitly state the unconditional purchase probability as

$$
\pi_{0}(p)= \begin{cases}1 & \text { if } p<\underline{p} \\ \dot{\pi}_{0}(p) & \text { if } \underline{p} \leq p \leq \bar{p} \\ 0 & \text { if } p>\bar{p}\end{cases}
$$


Author: Pricing When Customers Have Limited Attention

Note that the unconditional purchase probability $\pi_{0}(p)$ given in (11) is continuous in $p$. Hence, the conditional purchase probability $\pi_{i}(p)$ in $(7)$ is also continuous in $p$.

Given the belief $\alpha$, the expected revenue of the firm is given by $\mathcal{R}_{i}(p, \alpha)=p \times \pi_{i}(p)$, for $i \in\{H, L\}$. We denote the optimal price maximizing $\mathcal{R}_{i}(p, \alpha)$ by $p_{i}^{*}$, and the corresponding optimal revenue by $\mathcal{R}_{i}^{*}$.

Proposition 1 The optimal price $p_{i}^{*}, i \in\{H, L\}$, is such that $\underline{p} \leq p_{i}^{*} \leq \bar{p}$.

Proposition 1 shows that when customers are rationally inattentive and do not revise prior beliefs based on the observed price, the firm will never charge the true valuation of the product. The optimal price is always sandwiched between the two true quality values. This means that a high-quality firm has to lower its price since customers can never fully assess the true quality of the product. This naturally results in a loss for the high-quality firm. In sharp contrast, a low-quality firm benefits from limited attention since costly information acquisition and resulting inattention allows the firm to charge prices higher than dictated by the true nature of the product. ${ }^{6}$ Proposition 1 highlights the fact that due to customer inattention, the price range for the product is smaller than the true quality difference generating those prices, even in the absence of any competition. Evidently, customers' limited attention restrains the ability of the firm to differentiate its product in terms of quality and charge the appropriate premium. This is an important finding that will have a significant bearing on the equilibria under signalling.

Based on Proposition 1, to determine the optimal price $p_{i}^{*}$, it is sufficient to take

$$
\pi_{0}(p)=\dot{\pi}_{0}(p)=\frac{e^{p / \lambda}\left(e^{p / \lambda}-(1-\alpha) e^{q_{L} / \lambda}-\alpha e^{q_{H} / \lambda}\right)}{\left(e^{p / \lambda}-e^{q_{H} / \lambda}\right)\left(e^{p / \lambda}-e^{q_{L} / \lambda}\right)} .
$$

In theory, one could substitute $\pi_{0}(p)$ in (12) back into (7) and then optimize $\mathcal{R}_{i}(p, \alpha)$ over $\underline{p} \leq p \leq \bar{p}$. The extant literature suggests the revenue function is not concave for the standard MNL model of demand (Hanson and Martin 1996), implying the intractability of a price-based formulation. Song and Xue (2007) show that the revenue optimization problem becomes concave if market share, rather than product price, is used as the decision variable. Following this principle, from (7), we make the following variable change

$$
p\left(\pi_{i}\right)=q_{i}+\lambda \ln \left[\frac{\pi_{0}}{1-\pi_{0}} \times \frac{1-\pi_{i}}{\pi_{i}}\right], \quad i \in\{H, L\}
$$

\footnotetext{
${ }^{6}$ While intuitively appealing, this result fails to hold under the MNL choice used to model bounded rationality.
} 
and express the expected revenue of the firm as a function of $\pi_{i}$ as $\mathcal{R}_{i}\left(\pi_{i}\right)=p\left(\pi_{i}\right) \times \pi_{i}$. Let $\pi_{i}^{*}$ denote the optimal market share (conditional purchase probability) that maximizes $\mathcal{R}_{i}\left(\pi_{i}\right)$. Note that $\mathcal{R}_{i}\left(\pi_{i}^{*}\right)=$ $\mathcal{R}_{i}\left(p_{i}^{*}, \alpha\right)=\mathcal{R}_{i}^{*}$. The mapping between price and market share in (13) is well-defined when $\pi_{i} \in(0,1)$, i.e., the customer processes information to make the purchase decision. We also know that the firm achieves $\pi_{i}=1$ for all $p \leq \underline{p}$, Hence, the firm sets $p\left(\pi_{i}\right)=\underline{p}$ to maximize expected revenue. On the other hand, for $\pi_{i}=0$, since it does not matter what price the firm charges as $\mathcal{R}_{i}\left(\pi_{i}\right)=0$ for all $p \geq \bar{p}$, we take $p\left(\pi_{i}\right)=\bar{p}$. As a result, $\mathcal{R}_{i}\left(\pi_{i}\right)$ is continuous on $\pi_{i} \in[0,1]$, and there is a one-to-one mapping between optimal market share $\pi_{i}^{*}$ and optimal price $p_{i}^{*}$ (obtained through the function $\dot{\pi}_{0}(p)$ ).

The analysis of firm revenue through market share variables $\pi_{i}$ is still quite involved and arduous since $\pi_{0}$ in (13) is itself a function of $\pi_{H}$ and $\pi_{L}$ (as previously expressed in (8)). Nevertheless, we can prove the concavity of $\mathcal{R}_{i}\left(\pi_{i}\right)$ in $\pi_{i}$ for $i \in\{H, L\}$. Moreover, with the help of Proposition 1, we are able to establish that $\mathcal{R}_{H}(p, \alpha)$ is indeed concave in $p$, while $\mathcal{R}_{L}(p, \alpha)$ is decreasing and convex in $p$, over the relevant price interval $[\underline{p}, \bar{p}]$ for given $\alpha$. It turns out that both characterizations are required for our subsequent analysis.

Theorem $1(i) \mathcal{R}_{i}\left(\pi_{i}\right)$ is concave in $\pi_{i}$ for $i \in\{H, L\}$.

(ii) $\mathcal{R}_{H}(p, \alpha)$ is concave in $p ; \mathcal{R}_{L}(p, \alpha)$ is decreasing and convex in $p$ for $p \in[\underline{p}, \bar{p}]$.

From part (ii) of Theorem 1, we can immediately see that when the true quality nature of the product is low, it is optimal for the firm to charge a (low) price such that every customer purchases the product.

Corollary 1 When the product is low-quality, the optimal price $p_{L}^{*}$ and revenue $\mathcal{R}_{L}^{*}$ are given by:

$$
\mathcal{R}_{L}^{*}=p_{L}^{*}=\underline{p}
$$

When the true quality nature of the product is high, it is not possible to obtain the optimal price $p_{H}^{*}$ and optimal revenue $\mathcal{R}_{H}^{*}$ in a closed-form. Despite this complication, we are able to provide additional properties of the optimal price and revenue that shed light on the role of customer's limited attention.

Proposition 2 When the product is low quality, the optimal revenue $\mathcal{R}_{L}^{*}$ (and price $p_{L}^{*}$ ) is increasing in $\lambda$. On the other hand, when the product quality is high, the firm's optimal revenue $\mathcal{R}_{H}^{*}$ is non-monotone 
Author: Pricing When Customers Have Limited Attention

Article submitted to Management Science; manuscript no. MS-15-00653.R3

in $\lambda$. In particular, there exists a threshold $\lambda_{c}$ above which the firm's optimal revenue is increasing in $\lambda$.

As Proposition 2 elucidates, it is in the interest of a firm selling a low-quality product to customers with limited attention to make information acquisition more costly. The less questions asked by the customer, the less is revealed about the true (low) quality of the product, which in turn expedites the firm's ability to charge higher prices. Such firms would prefer to make product information more fuzzy and less revealing, and perhaps limit salesforce availability in order to take better advantage of limited attention of customers and costly information acquisition.

Intuitively one would expect the cost of information to play the opposite role for a high-quality product. Specifically, since a firm selling a high-quality product suffers from limited attention of the customers, it would be in the interest of the firm to make product quality information more accessible and as revealing as possible so that customers with limited attention can assess the true quality nature of the product with less effort. This intuition, however, is only partially correct. Increasing the cost of information $\lambda$ initially from relatively low levels results in customers asking less questions about the product and therefore learning less about the true (high) quality. In line with the intuitive thinking, this behaviour reduces the firm's profit. However, when $\lambda$ is sufficiently large, it becomes too costly for the customers to acquire and process more information about the product so they base purchasing decisions mostly on prior valuations. At this point, it does not matter for the firm whether the product is highquality or not - the pricing strategy of a high-quality product converges to that of a low-quality product, for which the optimal firm profit is increasing in $\lambda$. Hence, firm profit is not monotonic in $\lambda$. This result contrasts sharply with information search literature that finds prices to behave monotonically in the search costs (see Branco et al. 2012 for example).

The cost of information in our framework can also be viewed as a proxy for the intensity of limitations that customers face when attempting to obtain information about quality attributes of a product prior to purchase, which depends on the nature of the product itself. Academic literature distinguishes three types of products: search, experience, and credence. Search goods (Stigler 1961) are products where 
the customer can inspect the product and obtain relevant information prior to purchasing (relatively low information cost). Most commodities fall into this category. Experience goods (Nelson 1970) such as wine, restaurant services, have qualities that can be discovered only after full consumption (higher information costs). Credence goods (Darby and Karni 1973) like repair services, on the other hand, have qualities that cannot be evaluated, even during the time of consumption (very high information cost). Proposition 2 shows that for product/service categories where it is difficult to acquire information about the true quality, such as highly experiential or credence goods, it is better for the firm to take advantage of it (and making it even more difficult) regardless of what the true quality nature is. It also provides theoretical support for the argument that experts providing credence goods (e.g. doctors, mechanics) should charge a flat fee for a specific type of product/service regardless of the degree of complexity, essentially overcharging customers for small jobs (simple procedures) and undercharging for big jobs (elaborate procedures) (see Economist 2006). Dulleck and Kerschbamer (2006) reach this conclusion based on a model that features gaming opportunities for the firm, which can potentially take advantage of the customers in situations where the firm is not legally liable for the quality of the delivered product and the customer cannot verify the quality of the delivered product. Note that our result in Proposition 2 complements Dulleck and Kerschbamer (2006), as the aforementioned finding would still hold simply because of the inattentive nature of customers even when the firm does not have the potential to engage in any fraudulent behaviour.

The impact of remaining key parameters, prior beliefs $\alpha$ and relative quality level $q_{L}$, are more intuitive. As the prior belief that the product is of high-quality increases or as the true quality level of the low-quality product approaches to that of a high-quality product, the customers' expected valuation of the product given by $\alpha q_{H}+(1-\alpha) q_{L}$ also increases. Therefore, the optimal price, for both the lowand high-quality products, and the corresponding firm revenues are increasing in $\alpha$ and $q_{L}$. The next proposition formally establishes this monotonicity result.

Proposition 3 The optimal revenues $\mathcal{R}_{L}^{*}$ and $\mathcal{R}_{H}^{*}$, as well as the optimal prices $p_{L}^{*}$ and $p_{H}^{*}$ are increasing in $\alpha$ and $q_{L}$. 
Author: Pricing When Customers Have Limited Attention

Article submitted to Management Science; manuscript no. MS-15-00653.R3

\subsection{When Price Signals Quality}

We next explore the PBE pertaining to the signalling game between the firm and customers with limited inattention. Most signalling games have multiple equilibria since the PBE concept does not put restrictions on the belief structure of customers off the equilibrium path. This remains to be the case in our framework. Nevertheless, utilizing the central results of the previous section, we are able to provide a more refined characterization of the equilibria.

Theorem 2 The following are true for pure strategy PBE:

1. There does not exist a separating equilibrium.

2. A pooling equilibrium always exists. Specifically, for any $\hat{p}$ such that $\underline{p} \leq \hat{p} \leq \tilde{p}(\alpha):=$ $\left\{\max p: \mathcal{R}_{L}(p, \alpha) \geq q_{L}\right\}$ both types of firms charge the same price $\hat{p}$ with probability 1 (i.e., $\hat{\sigma}_{H}(p)=$ $\hat{\sigma}_{L}(p)=1$ if $p=\hat{p}$ and $\hat{\sigma}_{H}(p)=\hat{\sigma}_{L}(p)=0$ otherwise). This equilibrium is supported by the belief structure $\mu(p)=\alpha$ if $p=\hat{p}$ and $\mu(p)=0$ otherwise.

Theorem 2 substantiates the price convergence result obtained in the absence of signalling. Specifically, since a low-quality firm benefits from inattention while a high-quality firm suffers (c.f. Proposition 1), there is always the incentive for a low-quality firm to try to "pass" as a high-quality firm, rendering a quality-revealing separating equilibrium impossible. In contrast, a pooling equilibrium in which high- and low-quality firms charge the same price always exists. As in many other signalling games, this equilibrium can be obtained at a range of prices. This is simply due to the arbitrary nature of out-of-equilibrium beliefs. In particular, according to the pooling equilibria characterized in Theorem 2, the customer, upon observing an out-of-equilibrium price, forms the belief that the product is of low quality. In order to address the multiplicity of equilibria in signalling games, it is customary to apply refinement concepts that employ restrictions on out-of-equilibrium beliefs to rule out counter-intuitive equilibrium outcomes. As a result, the validity of equilibria, as well as the predictive power of the models, satisfying (surviving) the criterion are strengthened. Accordingly, the following proposition focuses on the pooling equilibria, and considers such a refinement on the beliefs (Theorem 2 already establishes the fact that a pure strategy separating equilibrium does not exist). 
Proposition 4 Suppose that for any out-of-equilibrium price $p, \mathcal{R}_{H}(p, \alpha)>\hat{\mathcal{R}}_{H}$ or $\mathcal{R}_{L}(p, \alpha)>\hat{\mathcal{R}}_{L}$ implies $\mu(p) \geq \alpha$, where $\hat{\mathcal{R}}_{H} \doteq \mathcal{R}_{H}\left(\hat{p}_{H}, \hat{\mu}\left(\hat{p}_{H}\right)\right)$ and $\hat{\mathcal{R}}_{L} \doteq \mathcal{R}_{L}\left(\hat{p}_{L}, \hat{\mu}\left(\hat{p}_{L}\right)\right)$. Then,

(i) For any $\lambda<\lambda_{c}$, there does not exist a pooling equilibrium.

(ii) For any $\lambda \geq \lambda_{c}$, there exists a unique pooling equilibrium: Both type of firms charge the same price $\hat{p}=\underline{p}$ with probability 1 (i.e., $\hat{\sigma}_{H}(p)=\hat{\sigma}_{L}(p)=1$ if $p=\hat{p}$ and $\hat{\sigma}_{H}(p)=\hat{\sigma}_{L}(p)=0$ otherwise.).

The refinement criterion in Proposition 4 means that any deviation to $p$ by either types of the firm is only profitable if the customer believes that at that price the probability that the product is of high quality is at least as high as the prior. This is not a very pessimistic belief, allowing deviations for high as well as low quality firms. But such deviations are associated with stronger beliefs about high quality.

Proposition 4 supports the key idea that information costs have a significant bearing on the equilibria. Under more reasonable off-equilibria beliefs, a pooling equilibrium only exists under high information costs. This affirms the notion that firms selling highly experiential or credence goods should charge a flat price regardless of the quality of the services rendered, even if customers interpret prices as signals of quality. Furthermore, the equilibrium price at sufficiently high information costs is exactly the same as in our model without signalling. From Proposition 2, this price is increasing in the cost of information. On the other hand, when the cost of information is low, this structure is no longer supported in equilibrium. In such environments, there is no pure strategy equilibrium.

In search for more insights for the low information case, we turn our attention to mixed strategies. An important candidate is the highly informative equilibrium that partially reveals quality levels (see Bester and Ritzberger 2001). In this semi-separating/pooling equilibrium, the high-quality firm plays a pure strategy and charges a high price, while the low-quality firm adopts a mixed strategy by randomizing between imitating the high-quality firm's price and revealing its low-quality by charging $q_{L}$. Let $f>0$ denote the probability that the low-quality firm imitates the high-quality firm price. This semi-separating/pooling PBE in terms of $\{\hat{\sigma}, \hat{\pi}, \hat{\mu}, \hat{f}\}$ can be specified for any $\hat{f}>0$ as:

$$
\hat{\sigma}_{H}(p)=1 \text { if } p=\hat{p}_{H}=\tilde{p}(\hat{\mu}) \quad(\text { as previously defined in Theorem 2) }
$$


Author: Pricing When Customers Have Limited Attention

$$
\begin{aligned}
& \hat{\sigma}_{L}(p)= \begin{cases}\hat{f} & \text { if } p=\hat{p}_{H} \\
1-\hat{f} & \text { if } p=q_{L}\end{cases} \\
& \hat{\pi}_{H}(p)=\pi_{H}^{\hat{\mu}}(p) \quad \hat{\pi}_{L}(p)=\pi_{L}^{\hat{\mu}}(p) \\
& \hat{\mu}(p)= \begin{cases}\frac{\alpha}{\alpha+(1-\alpha) \hat{f}} & \text { if } p=\hat{p}_{H} \\
0 & \text { otherwise }\end{cases}
\end{aligned}
$$

Under this equilibrium, when customers observe the low price $q_{L}$, they believe (perfectly) that the product is low-quality. On the other hand, when they observe the high price $\hat{p}_{H}>q_{L}$, they form interim beliefs that the product is high-quality according to the Bayes' Rule $(\hat{\mu}=\alpha /(\alpha+(1-\alpha) \hat{f})$. Hence, they need to acquire and process additional information to distinguish the true quality of the product. As before, customers optimally acquire and process quality information, and make choices consistent with their updated beliefs. Recall that by definition $\mathcal{R}_{L}\left(\hat{p}_{H}, \hat{\mu}\right)=q_{L}$ which means that at the high price $\hat{p}_{H}=\tilde{p}(\hat{\mu})$, the low-quality firm is indifferent to imitating the high price or separating itself by charging the low price $q_{L}$ (hence firm optimality is satisfied). Charging this high price is optimal for the high-quality firm as well because otherwise customers believe that the quality is low (which would render lower profits).

It can be easily shown that for any fixed $\lambda>0$, the equilibrium high price $\hat{p}_{H}$ is decreasing in the equilibrium imitation rate $\hat{f}$. Hence as the imitation rate of the low-quality firm decreases, the equilibrium shifts to a more "separating" structure with the high-price increasing towards the true quality level $q_{H}$. In contrast, when the imitation rate of low-quality firm is higher, the equilibrium shifts towards a more "pooling" structure with more converged prices for high and low quality firms. This is rather intuitive and perhaps it would be more interesting to establish such a result when the cost of information and equilibrium imitation rates strategically interact. This is not the case in the above game; both type of firms decide on prices, and the equilibrium is specified for given imitation rates and cost of information. Such interactions can be present if the pricing and imitation rates are determined by the high and low quality firms respectively. This makes sense since the low-quality firm's only equilibrium action is to imitate the price set by the high-quality firm. To this end, consider a slight variation to the above game, in which the high-quality firm strategy remains as pricing, while 
the low-quality firm strategy is the imitation rate. ${ }^{7}$ The equilibrium $\{\hat{\sigma}, \hat{\pi}, \hat{\mu}, \hat{f}\}$ of this game is similar to the previous case, only that $\hat{p}_{H}$ and $\hat{f}$ would be chosen to maximize high-quality and low-quality firm profits respectively:

$$
\begin{array}{rlr}
\hat{\sigma}_{H}(p)=1 \text { if } p=\hat{p}_{H}=\underset{q_{L} \leq p \leq q_{H}}{\arg \max } \mathcal{R}_{H}(p, \hat{\mu}) & \hat{\sigma}_{L}(p)= \begin{cases}\hat{f} & \text { if } p=\hat{p}_{H} \\
1-\hat{f} & \text { if } p=q_{L}\end{cases} \\
\hat{\mu}(p)= \begin{cases}\hat{\pi}_{H}(p)=\pi_{H}^{\hat{\mu}}(p) & \hat{\pi}_{L}(p)=\pi_{L}^{\hat{\mu}}(p) \\
0 & \text { if } p>q_{L}\end{cases} & \hat{f}=\underset{0 \leq f \leq 1}{\arg \max }\left\{f \mathcal{R}_{L}\left(\hat{p}_{H}, \hat{\mu}\right)+(1-f) q_{L}\right\}
\end{array}
$$

As before, when customers observe the low price $q_{L}$, they believe (perfectly) that the product is lowquality. For any other price $\hat{p}_{H}>q_{L}$, they form interim beliefs that the product is high-quality as $(\hat{\mu}=\alpha /(\alpha+(1-\alpha) \hat{f})$ and make optimal choice according to GMNL, consistent with this belief. The high-quality firm chooses the (high) price to maximizes his profit under the equilibrium belief structure. In a similar vein, at the equilibrium $\hat{f}$, the low-quality firm profit is maximized.

Notice that as $\lambda \downarrow 0$, customers can determine actual quality very easily regardless of their prior beliefs, implying $\hat{p}_{H}$ will approach $q_{H}$. Since imitating this price would be very costly to the low-quality firm, we would expect $\hat{f}$ to approach zero, and the equilibrium to converge to the perfect information (separating) equilibrium. In contrast, when $\lambda$ is sufficiently large (and in particular $\lambda \geq \lambda_{c}$,), $\hat{p}_{H}$ will be very close to low-quality firm's optimal price $\arg \max _{q_{L} \leq p \leq q_{H}} \mathcal{R}_{L}(p, \hat{\mu})$. Hence, we would expect the low-quality firm to prefer imitating the high-quality firm price $(\hat{f}=1)$, and making the equilibrium identical to the pooling equilibrium.

It can be checked easily that the low-quality firm's profit (see RHS of expression (21)) is not necessarily unimodal in $f$ and $\hat{f}$ can take extreme values $\{0,1\}$ or an intermediate value. Hence, although an equilibrium always exists, it is not possible to characterize its properties analytically. We conduct numerical experiments to explore the behavior of the equilibrium. Specifically, we generate problem settings by systematically varying a number of key parameters of a base scenario. In the base scenario, ${ }^{7}$ Since the low-quality firm is deciding only on how much it should imitate the high-quality firm price, we can also allow no imitation $(f=0)$ in his strategy space for this game. 
Author: Pricing When Customers Have Limited Attention

we set the levels of the high-quality and low-quality as $q_{H}=10$ and $q_{L}=5$, respectively. The customer's prior belief is such that the product is equally likely to be either high- or low-quality $(\alpha=0.5)$ and the unit cost of information is $2(\lambda=2)$. Figures 2 and 3 depict the impact of unit cost of information $(\lambda)$, prior belief of customers $(\alpha)$, and the low-quality level $\left(q_{L}\right)$ on the equilibrium imitation rate and the equilibrium price of the product, respectively, depending on the product's true quality level. We note that the equilibrium revenues follow similar patterns to the corresponding prices and hence are omitted.

Our numerical experiments show that the above intuition regarding information costs holds. Specifically, as seen in Figure 2(a), when $\lambda$ is low, $\hat{f}=0$ (denoted as PIE on the figures). For intermediate $\lambda$ levels, the strict semi-separating/pooling equilibrium (denoted as $\mathrm{S} / \mathrm{PE}$ on the figures) emerges $(0<\hat{f}<1)$, while for high $\lambda$ levels, ${ }^{8} \hat{f}=1$ (denoted as PE on the figures). Overall, the imitation rate of the high price of the high-quality firm increases as information becomes more costly. The price (and revenue) implications ensue, as seen in Figure 3(a). When $\lambda$ is low, equilibrium prices reveal true qualities, $\hat{p}_{H}=10$ and $\hat{p}_{L}=5$. On the other extreme, when $\lambda$ is high, both high- and low-quality firms charge the same price, which converge to the customer's expected valuation for the product $\alpha q_{H}+(1-\alpha) q_{L}=7.5$, as $\lambda \uparrow \infty$. Observe that as the cost of information $\lambda$ increases, $\hat{p}_{L}$ generally increases (cf. Proposition 2). In contrast, $\hat{p}_{H}$ does not decrease monotonically; it is first decreasing but then after a threshold, the PE prevails and it starts increasing. As the equilibrium revenues mirror prices, these results are consistent with those obtained in the absence of signalling (cf. Proposition 2).

Customer's prior belief about the quality of the product also has a profound impact on the equilibrium prices (and revenues), as evident from Figure 3(b). When the customer's prior belief is relatively strong ( $\alpha \downarrow 0$ or $\alpha \uparrow 1$ ), she has to spend more effort in order to determine the actual quality level, which leads to converged prices and PE emerges, as in Figure 2(b). Accordingly, in such cases, product price does not signal quality - as $\alpha \downarrow 0$, a high-quality product is priced as if its quality is low to be able to sell the product, and as $\alpha \uparrow 1$, a low-quality product commands the same price of a high-quality product since customers are absolutely wrong in their initial beliefs about the product's true quality nature. When the beliefs are less strong, (moderate $\alpha$ ), customers are more easily able to glean quality ${ }^{8}$ In this example, $\hat{f}=1$ for any $\lambda \geq 2.3$. The critical $\lambda_{c}=2.43$. 
Author: Pricing When Customers Have Limited Attention Article submitted to Management Science; manuscript no. MS-15-00653.R3

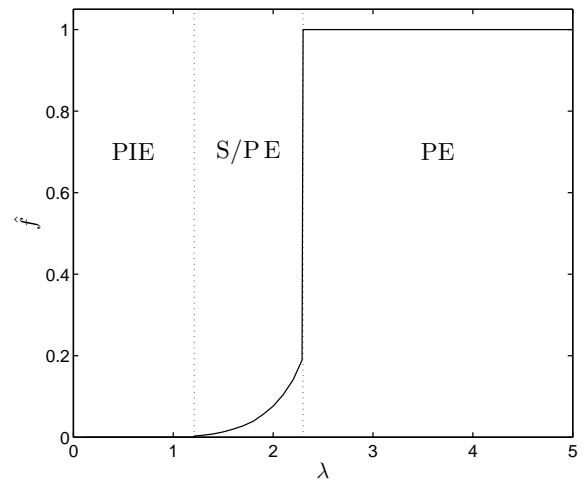

(a)

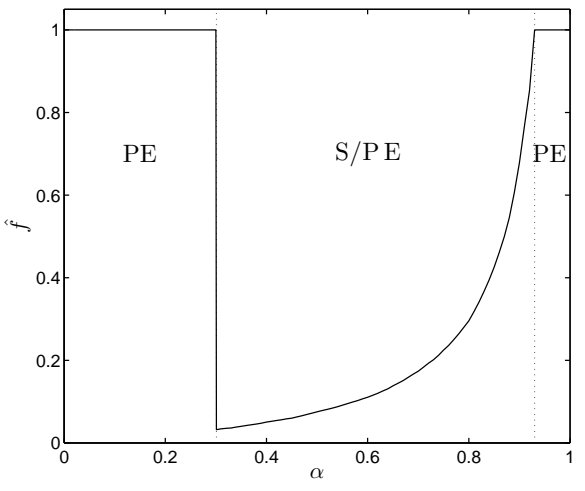

(b)

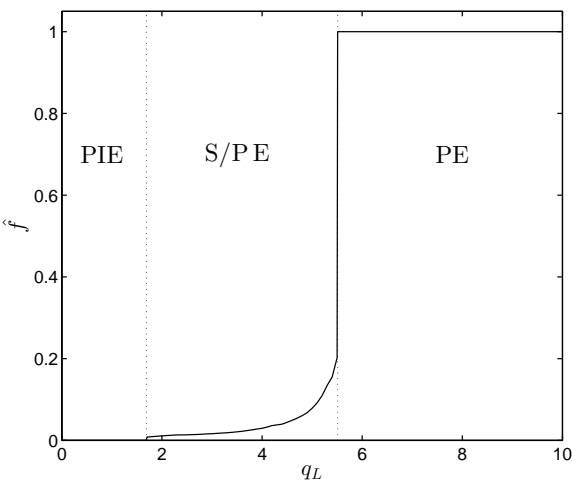

(c)

Figure 2 Impact of $\lambda$ on Perfect Information (PIE), Semi Separating/Pooling (S/P E) and Pooling (PE) Equilibria $\hat{f}$

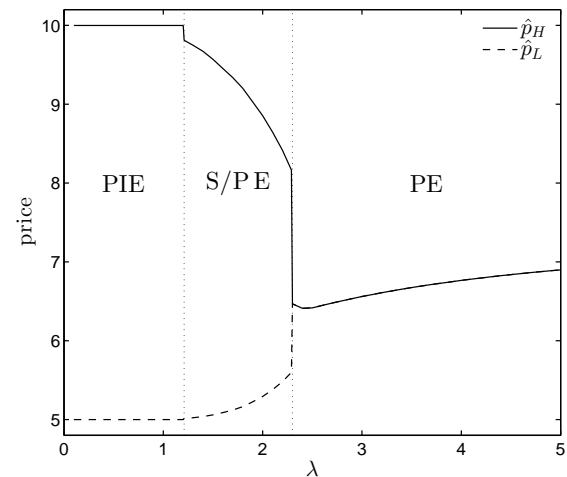

(a)

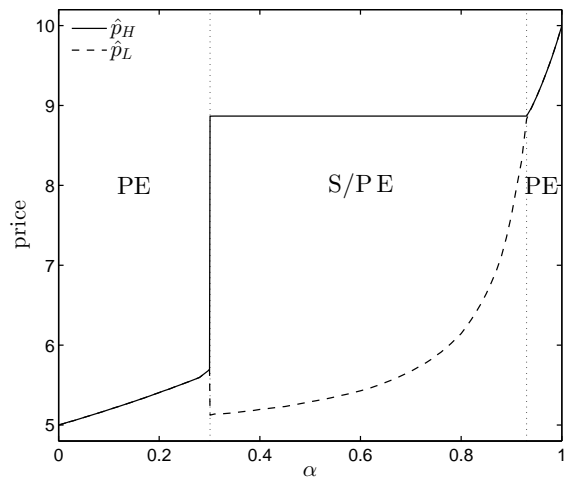

(b)

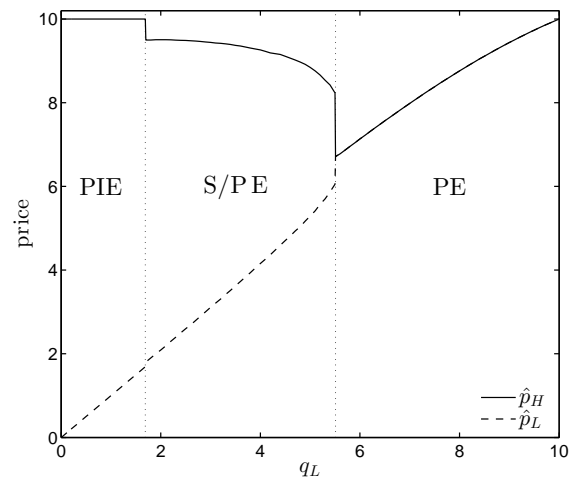

(c)

Figure 3 Impact of $\lambda$ on Perfect Information (PIE), Semi Separating/Pooling (S/P E) and Pooling (PE) Equilibria prices

by acquiring information, and in turn we observe differentiated prices in equilibrium. Also, note from Figure 3(b) that equilibrium prices are generally increasing in $\alpha$, which conform with the results of the previous section (cf. Proposition 3). Clearly, a low-quality product increasingly benefits from quality misjudgments, while a high-quality product naturally benefits from more accurate assessment of its quality by the customers.

Figures 2(c) and 3(c) depict the impact of $q_{L}$ on the equilibrium imitation rate and prices. As $q_{L}$ increases, the true quality level of the low-quality product approaches to that of a high-quality product, essentially reducing the perceived degree of product differentiation. Note that when $q_{L}$ is sufficiently lower than $q_{H}$, inattentive customers face a potentially considerable quality risk. Therefore, they are more willing to gather and process information about the true quality at some cost, reducing the tendency for price convergence in equilibrium. For these cases, either the separating equilibrium is 
Author: Pricing When Customers Have Limited Attention

observed $(\hat{f}=0)$, or the low-quality firm imitates the price of the high-quality firm at a certain rate $(0<\hat{f}<1)$. Furthermore, $\hat{p}_{L}$ steadily increases with $q_{L}$ in this range, as expected (cf. Proposition 3$)$. The high-quality firm price, on the other hand, suffers from an increase in $q_{L}$ initially. This is because as the low-quality firm imitates the high-quality price, it becomes increasingly difficult for customers to differentiate the products, which curbs the ability of the high-quality firm to reflect the product's true quality level in its price. In contrast, when $q_{L}$ is sufficiently close to $q_{H}$, then the quality levels are so close that it becomes prohibitively expensive for customers to ask enough number of questions to differentiate between a high- or a low-quality product. Consequently, PE ensues and customers purchase without processing any information. This price, which is the same regardless of the true quality level, continues to increase with the quality level $q_{L}$. Put differently, lack of significant quality distinction curbs the ability for price differentiation when customers are rationally inattentive.

\section{Heterogeneous Customers}

Thus far we have assumed that customers are homogenous; (i) they are equally informed about product quality and have identical information costs, and (ii) they share the same prior belief about product quality. We next incorporate customer heterogeneity by relaxing these assumptions.

\subsection{Heterogeneity in Information Costs}

Suppose that, as in Debo et al. (2012), customers are heterogeneously informed about the quality, such that only a fraction of them are uninformed about quality, while others are fully informed (and hence know the true quality of the product). Equivalently, we can consider the market to be heterogenous in information costs, consisting of both inattentive customers with cost of information of $\lambda>0$ and perfectly attentive customers with zero information cost. Let the fraction of inattentive customers be $\beta$. Inattentive customers update their prior $\alpha$ based on price $p$, form their interim belief $\mu(p)$, and purchase the product of quality $q_{i}$ with probability $\pi_{i}^{\mu}(p)$ as before. On the other hand, attentive customers purchase the product only if $p \leq q_{i}$. In this case, the expected profit can be written as

$$
\mathcal{R}_{i}(p, \mu(p))=\beta p \pi_{i}^{\mu}(p)+(1-\beta) p 1_{p \leq q_{i}} \text { for } i \in\{H, L\},
$$

where $1_{x}$ is the indicator function. Customer heterogeneity brings forth new trade-offs for the firm in choosing the optimal product price. Clearly, the firm has to decide which segments of the market 
to serve in setting its price when customers are heterogenous. To develop an understanding of this, we first focus on the case of no signalling, i.e. $\mu(p)=\alpha$. Dropping $\mu$ from the notation as in $\S 3.2$, we characterize the optimal strategy as follows.

Proposition 5 When prices do not signal quality, the optimal pricing strategy under information cost heterogeneity is as follows:

1. When the product quality is high, there exists a threshold $\underline{\beta}=\frac{q_{H}-\tilde{p}_{H}}{q_{H}-\left(1-\pi\left(\tilde{p}_{H}\right)\right) \tilde{p}_{H}}$ such that for any $\beta \geq \underline{\beta}$, the firm serves both market segments with price $\check{p}_{H}$, which uniquely maximizes $\mathcal{R}_{H}(p, \alpha)=$ $\beta p \pi_{H}(p)+(1-\beta) p$. If $\beta<\underline{\beta}$, the firm serves attentive customers only with price $q_{H}$.

2. When the product quality is low, there exists a threshold $\bar{\beta}=\frac{q_{L}}{q_{L}+q_{H}-\lambda \ln \left[(1-\alpha) e^{q_{H} / \lambda}+\alpha e^{q_{L} / \lambda}\right]}$ such that for any $\beta \leq \bar{\beta}$, the firm serves both market segments with price $q_{L}$. If $\beta>\bar{\beta}$, the firm serves inattentive customers only with price $p_{L}^{*}=q_{L}+q_{H}-\lambda\left[(1-\alpha) e^{q_{H} / \lambda}+\alpha e^{q_{L} / \lambda}\right]$.

Evidently, the optimal pricing strategy is more complicated when customers are heterogenous in quality information or information costs. Nevertheless, the effects of heterogeneity are rather reasonable - the higher the fraction of informed/attentive customers (lower $\beta$ ), the closer the prices to the true quality levels of the products. Naturally, a high-quality firm benefits from an increased proportion of informed/attentive customers while a low-quality firm loses from it. We also observe that heterogeneity does not lead to an unexpected price ordering between high- and low-quality products (this contrasts with Debo et al. (2012) which shows that a high-quality firm can choose a slower rate of service due to heterogeneity). It is indeed never optimal for the firm to set the price of the low-quality product higher than that of the high-quality product. Likewise, a low-quality product never garners higher profits for the firm than a high-quality product.

We now consider the case when prices signal quality, and focus on the firm's PBE in which the high-quality firm chooses the optimal price, whereas the low-quality firm sets the imitation rate of this particular price, as characterized in (19)-(21). Figure 4 illustrates the impact of the level of heterogeneity in information costs on the equilibrium imitation rate $\hat{f}$ and prices $\hat{p}_{H}$ and $\hat{p}_{L}$. As $\beta$ decreases, the ratio of attentive customers increase and in turn the effect of signalling is expected to decrease. Accordingly, 
for our base scenario, we notice in Figure 4(a) that the pooling equilibrium when $\beta=0.9$ is observed at higher information costs compared to the case when $\beta=1$ (corresponding to the homogenous case). When $\beta=0.8$, the pooling equilibrium does not even arise for the depicted range of information cost values. Further, from Proposition 5, we know that if $\beta$ is less than a certain threshold, the highquality firm has the incentive to only serve the attentive customers, eliminating the effect of signalling altogether. In line with this, when prices signal quality, if $\beta \leq 0.51$, only the perfect information equilibrium is observed.

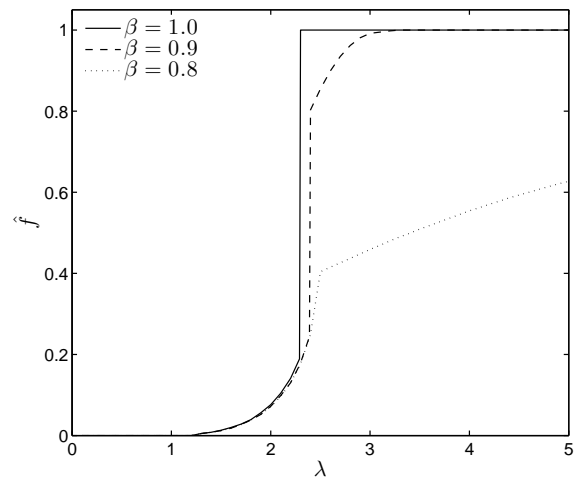

(a)

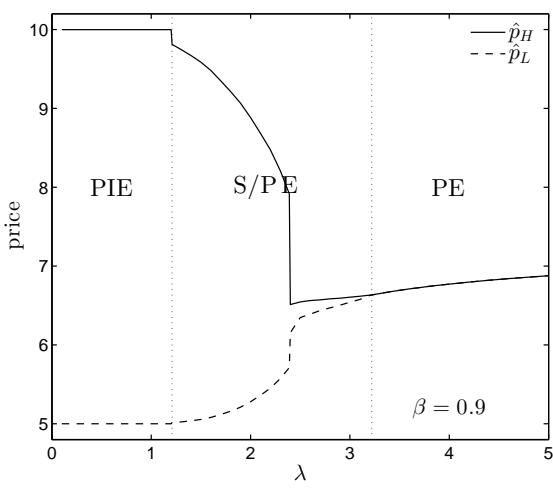

(b)

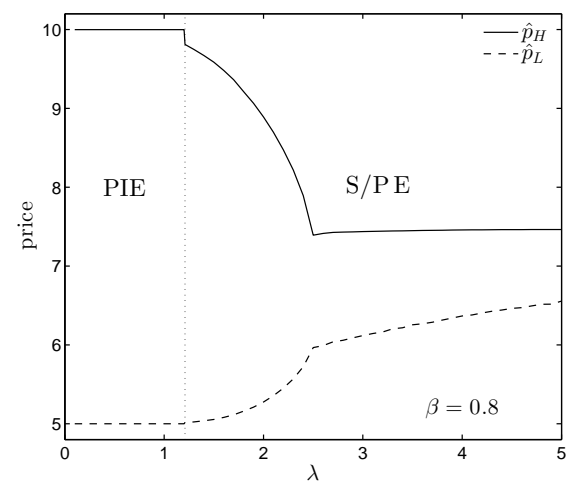

(c)

Figure $4 \quad$ Impact of heterogeneity in information costs

\subsection{Heterogeneity in Prior Beliefs}

Suppose now that customers have heterogeneous priors about product quality - a $\gamma$ fraction of customers believe that the product is high-quality with probability $\alpha_{1}$ (or expect the quality to be $\alpha_{1} q_{H}+\left(1-\alpha_{1}\right) q_{L}$ ), whereas the remaining $1-\gamma$ fraction believe this said probability is $\alpha_{2}$ (or expect the quality to be $\left.\alpha_{2} q_{H}+\left(1-\alpha_{2}\right) q_{L}\right)$. Here we assume $\alpha_{1}>\alpha_{2}$ without loss of generality. Customers (segments) first update their respective priors, and form interim beliefs $\mu_{1}(p)$ and $\mu_{2}(p)$ consistent with the Bayes' rule. Then each customer selects her information strategy optimally, forming posterior beliefs that are consistent with her own interim belief. Accordingly, we can express the firm's profit as follows

$$
\mathcal{R}_{i}\left(p, \mu_{1}(p), \mu_{2}(p)\right)=\gamma p \pi_{i, 1}^{\mu}(p)+(1-\gamma) p \pi_{i, 2}^{\mu}(p) \text { for } i \in\{H, L\}
$$


where $\pi_{i, k}^{\mu}(p)$ is the conditional purchase probability of the product with quality $q_{i}, i \in\{H, L\}$ by customers with belief $\mu_{k}(p), k \in\{1,2\}$. As in $\S 4.1$, we first assume $\mu_{1}(p)=\mu_{2}(p)=\alpha$ and study the case when the product price does not signal its quality.

Proposition 6 When prices do not signal quality, the optimal pricing strategy under heterogeneity in prior beliefs is as follows:

1. When the product quality is high, the optimal price $p_{H}^{*}$ can be uniquely determined since $\mathcal{R}_{H}(p, \alpha)$ is piecewise concave in $p$.

2. When the product quality is low, there exists a threshold $\underline{\gamma}=\frac{q_{L}+q_{H}-\lambda \ln \left[\left(1-\alpha_{2}\right) e^{q_{H} / \lambda}+\alpha_{2} e^{q_{L} / \lambda}\right]}{q_{L}+q_{H}-\lambda \ln \left[\left(1-\alpha_{1}\right) e^{q_{H} / \lambda}+\alpha_{1} e^{q_{L} / \lambda}\right]}$ such that for any $\gamma \geq \underline{\gamma}$, the firm sets the price as $p_{L}^{*}=q_{L}+q_{H}-\lambda\left[\left(1-\alpha_{1}\right) e^{q_{H} / \lambda}+\alpha_{1} e^{q_{L} / \lambda}\right]$; otherwise the firm sets the price lower as $p_{L}^{*}=q_{L}+q_{H}-\lambda\left[\left(1-\alpha_{2}\right) e^{q_{H} / \lambda}+\alpha_{2} e^{q_{L} / \lambda}\right]$.

Proposition 6 states that in pricing the high-quality product, the firm essentially considers the blended response from both customer segments, whereas in pricing the low-quality product, it merely decides whether to serve both segments or to serve the segment with the higher quality expectation only. In the particular signalling game that we also considered in $§ 4.1$, the low-quality firm chooses the rate at which it would imitate the optimal price of the high-quality firm (versus setting price at $\left.q_{L}\right)$. Therefore, we expect the overall behaviour of the equilibrium imitation rates and prices, when customers are heterogenous in their prior beliefs, to be very similar to what we previously depicted in Figures 2(a) and 3(a). Indeed, for the base scenario with $\alpha_{1}=0.75, \alpha_{2}=0.25$, and $\gamma=0.5$, we observe in Figure 5(a) that as in the homogenous case $\left(\alpha_{1}=\alpha_{2}=0.5\right)$, when $\lambda$ is low, $\hat{f}=0$ and the equilibrium is separating. On the other hand, when $\lambda$ is high, $\hat{f}=1$ and signalling induces the pooling of prices (see also Figure 5(b)). At intermediate levels of $\lambda$, we observe $0<\hat{f}<1$ and the semi separating/pooling equilibrium ensues.

\section{Discussion and Concluding Remarks}

In this paper, we develop a framework to analyze pricing decisions in the presence of customers with limited attention. We consider a scenario where customers are ex-ante unable to assess the exact "quality" aspect of a product, but can observe the price, which can signal the quality of the product. 


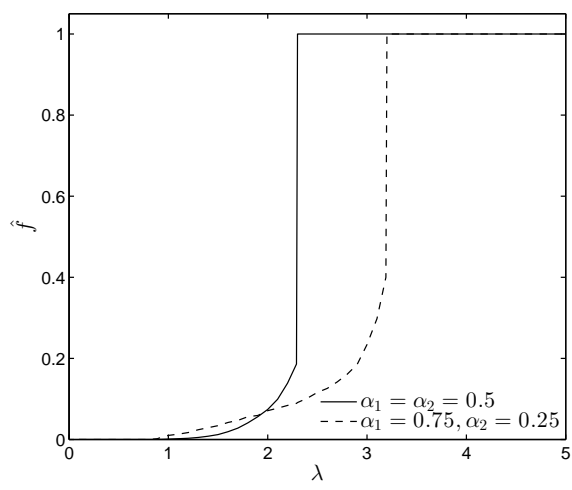

(a)

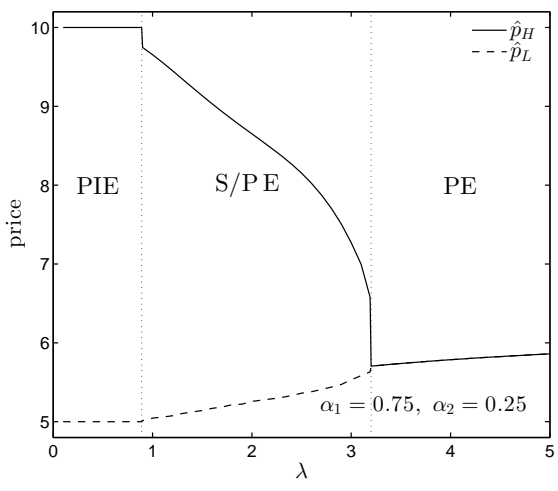

(b)

Figure 5 Impact of heterogeneity in prior beliefs

In accordance with the theory of rational inattention, the customers in our model can attend to information regarding the quality of the product at some cost, and make the attention allocation decisions optimally. The firm, on the other hand, has more experience with the product, and knows its exact quality. Anticipating rationally inattentive customer behaviour (which is shown in the literature to generate conditional purchase probabilities that follow a generalized multinomial logit form) and the resulting demand, the firm selects the price to maximize its revenue.

Our model provides a realistic representation of limited customer attention and fits most nonfunctional product (goods or services) markets where customers do not purchase the product at very high frequencies, and hence pay attention to the posted prices of the distinct choices. It is also general in that it incorporates signalling of quality via prices and customer heterogeneity, offers unifying interpretations across multiple product types (search, experience and credence goods) via its stylized costly information acquisition and processing aspect, and has connections to the quantal choice models of bounded rationality as well as costly information search models.

Through analytical characterizations and complementary numerical experiments, we provide a full account of optimal prices and firm revenues in the presence of customers with limited attention, and show how they are shaped by (i) ease of obtaining information, (ii) prior beliefs about product qualities, and (iii) relative quality levels of the products, in different settings with and without signalling. In order to avoid repetition, instead of providing a summary of these results, we discuss here the prescriptive results and managerial insights generated therefrom, together with practical examples. We can group 
these insights in two categories: information provision (how easy/difficult should the firm make the access of customers to quality revealing information) and product positioning (how should the firm try to shape the quality perceptions of the customers). ${ }^{9}$

We show that when the cost of information is relatively low (search goods such as sports equipment, cameras, LED TVs), it is always in the interest of a firm selling a low-quality product to make it difficult for customers to gather information about its product. We see examples of this in practice too. For example, many knock-off smartphone and tablet producers do not even maintain a proper website to inform customers about their (cheap) imitation products. A firm with a high-quality product, on the other hand, should do just the opposite. This can be done by proactive targeted advertising, salesforce, providing demo/trial models etc. Samsung for example employs own sales associates within Best Buy stores to inform customers about its high-end TVs. In contrast, when the cost of information is sufficiently high (credence or highly experiential good such as medical services, wine), the information provision preferences do not depend on true quality levels; regardless of the true quality of the product, the firm should make it even more difficult for the customers to assess quality.

The fundamental result that drives the firm's information provision preference is the fact that as the cost of information increases, the optimal price of high- and low-quality products converge. This is because when it is difficult for customers to attend to quality revealing information, they start to ignore it by making choice decisions mostly based on prior beliefs. Knowing this, firms with high- and low-quality products are prompted to charge the same price. This result provides alternative support as to why in unregulated markets prices of credence goods should converge. It is also robust as it holds when prices signal quality to customers as well as when prices do not signal any quality information.

Our model and results offer insights as to how a firm should try to influence quality perceptions and position its product accordingly. There are two fundamental ways the firm can do this in our framework: It can try to improve the perceived quality level of its product or it can try to improve the belief that it is offering the superior ("higher" quality) product. We show that the latter strategy is a

\footnotetext{
${ }^{9}$ Needless to say, these actions might bear some costs on behalf of the firm, which are not considered here. In this regard, we are only talking about the benefits to the firm, which should be traded-off against potential costs (if any).
} 
Author: Pricing When Customers Have Limited Attention

safe one since it improves firm profits even for a firm with a low-quality product. It is effective even when the quality levels of the products are similar. Improving the perceived quality of the product can also be effective, if it is increasing the relative quality distinction. Otherwise, it mostly benefits the firm selling a low-quality product.

We find that market heterogeneity in information costs can have a compounding effect in both information provision and product positioning strategies. Since a firm selling a high-quality product suffers from the inattention of customers, it should proactively try to enhance awareness of its highquality (increase the size of more informed customers), by allowing pre-launch screenings (as in movies), advertising, social marketing, or through other means. Naturally, the reverse is true for a firm selling a low-quality product. Heterogeneity in customer's prior belief about quality, however, does not have a major impact on the qualitative insights.

It is important to note that our insights may depend on the single-firm, monopolistic market structure assumed in the paper. In recognition of this fact, we have studied a limited and highly stylized extension of our model (with two single-product firms and no signalling) to explore the economic incentives of competing firms serving customers with limited attention. Indeed, our preliminary results demonstrate that when information costs are high, competition may have an impact on both information provisioning and product positioning. Specifically, in sharp contrast to the case where the firm enjoys a dominating position in the market, when there are distinct competing products in the market, it might be better for the firm to make it easier for customers to acquire quality revealing information. The benefit of increasing the customer's ability to distinguish the products is the softening of price competition, which is already amplified due to difficulties in learning the true qualities of the products. Hence, while a restaurant offering only one choice of wine may deliberately not reveal much information about it (and ambiguously refer to it as the "house wine"), it is in the interest of wineries in a wine-growing region to offer tours (as they often do), enabling the customers to sample the lower quality wines along with high-quality ones. Regarding product positioning, we find that attempting to improve the quality perception of a low-quality product might backfire under competition when information costs are high, as this would intensify competition. In such settings, it might be better for the low-quality 
firm to reduce its perceived quality level, improving the profitability of both firms. A recent example of a company which has gone through this experience is Mulberry, a producer of (luxury) ladies leather handbags. After failing to push its brand upmarket with increased prices, it repositioned itself on the lower luxury market by promoting "affordable luxury" (Financial Times 2014). Improving the customer beliefs about high quality, on the other hand, remains to be beneficial to both firms under competition. Hence, instead of trying to improve the quality perceptions of the consumers, the firm might be better off by simply pitching to its customers that it is offering a "superior" product. These initial insights highlight the fact that a comprehensive analysis of competition would be an interesting direction for future research.

We conclude with two remarks. The first one pertains to alternative pricing strategies. Our paper establishes the existence and some key properties of the optimal pricing strategy of a firm selling to rationally inattentive customers, but computing this optimal price may require considerable effort on behalf of the firm. There are natural alternative pricing schemes that do not depend on the cost of information and are easier to compute, as well as those that are derived from alternative specifications of the consumer choice process. We have conducted numerical studies to test the relative performance of a number of pricing strategies, including MNL-based pricing as well as (sequential) search pricing discussed in $§ 3.1$, and observed significant profit gaps and high variability in the performance. These signify that overlooking the inattentiveness of customers in pricing can have significant impact on profitability. The second remark relates to product assortment strategies. Limited attention has implications on choice and optimal prices, which in turn can have significant bearing on the assortment decisions of the firm. In particular, "less" can be "more" when customers are constrained in acquiring and processing information. We confirm this intuition in a preliminary model with two products by identifying a setting where adding a new product to an existing assortment can actually reduce profits. Determining the optimal assortment of the firm (with or without pricing), however, remains to be a challenging task. This is because it might be easier to obtain information about some products than about others, by the very nature of the product or by the fact that they are readily available for "touch and feel" compared to others that are not available for such an experience. This calls for a generalized 
Author: Pricing When Customers Have Limited Attention

Article submitted to Management Science; manuscript no. MS-15-00653.R3

choice model that allows the information cost to vary among options. Further, this generalized choice model has to be embedded in assortment optimization, which is known to be difficult to solve even for simpler choice models like MNL or nested-MNL (they are often NP-hard or NP-complete). These are subjects of our ongoing research.

\section{References}

Akerlof, G. A. 1970. The market for" lemons": Quality uncertainty and the market mechanism. The quarterly journal of economics $488-500$.

Aksoy-Pierson, M., G. Allon, A. Federgruen. 2013. Price competition under mixed multinomial logit demand functions. Management Science 59(8) 1817-1835.

Bagwell, K., M. H. Riordan. 1991. High and declining prices signal product quality. The American Economic Review 81(1) 224-239.

Bester, H. 1998. Quality uncertainty mitigates product differentiation. The RAND Journal of Economics $828-844$.

Bester, H., K. Ritzberger. 2001. Strategic pricing, signalling, and costly information acquisition. International Journal of Industrial Organization 19(9) 1347-1361.

Branco, F., M. Sun, J. M. Villas-Boas. 2012. Optimal search for product information. Management Science 58(11) 2037-2056.

Caplin, A., D. Martin. 2014. A testable theory of imperfect perception. The Economic Journal 125(February) $184-202$.

Chen, Y., X. Su, Xi. Zhao. 2012. Modeling bounded rationality in capacity allocation games with the quantal response equilibrium. Management Science 58(10) 1952-1962.

Darby, M. R., E. Karni. 1973. Free competition and the optimal amount of fraud. Journal of Law Es Economics $1667-88$.

Debo, L.G., C. Parlour, U. Rajan. 2012. Signaling quality via queues. Management Science 58(5) 876-891.

Debo, L.G., S. Veeraraghavan. 2014. Equilibrium in queues under unknown service times and service value. Operations Research 62(1) 38-57. 
Dulleck, U., R. Kerschbamer. 2006. On doctors, mechanics, and computer specialists: The economics of credence goods. Journal of Economic Literature 44(1) 5-42.

Economist. 2006. Economics focus: Sawbones, cowboys and cheats. The Economist April 15, 2006.

Erdem, T., M. P. Keane, B. Sun. 2008. A dynamic model of brand choice when price and advertising signal product quality. Marketing Science 27(6) 1111-1125.

Financial Times. 2014. Mulberry aims for "affordable luxury" after failed move upmarket. Financial Times

\section{April 17, 2014.}

Hanson, W., K. Martin. 1996. Optimizing multinomial logit profit functions. Management Science 42(7) 992-1003.

Luce, R.D. 1959. Individual Choice Behavior: A Theoretical Analysis. Wiley, New York.

Mackowiak, B., M. Wiederholt. 2009. Optimal sticky prices under rational inattention. American Economic Review 99(3) 769-803.

Manzini, P., M. Mariotti. 2014. Stochastic choice and consideration sets. Econometrica 82(3) 1153-1176.

Martin, Daniel. 2016. Strategic pricing and rational inattention to quality. Working Paper. Available at SSRN 2393037.

Matějka, F. 2015. Rigid pricing and rationally inattentive consumer. Journal of Economic Theory 158(B) $656-678$.

Matějka, F. 2016. Rationally inattentive seller: Sales and discrete pricing. The Review of Economic Studies 83(3) $1125-1155$.

Matějka, F., A. McKay. 2012. Simple market equilibria with rationally inattentive consumers. American Economic Review 102(3) 24-29.

Matějka, F., A. McKay. 2015. Rational inattention to discrete choices: A new foundation for the multinomial logit model. American Economic Review 105(1) 272-298.

McKelvey, R. D., T. R. Palfrey. 1995. Quantal response equilibria for normal form games. Games and economic behavior 10(1) 6-38.

McKowiak, B., M. Wiederholt. 2015. Business cycle dynamics under rational inattention. Review of Economic Studies. Forthcoming. 
Author: Pricing When Customers Have Limited Attention

Nelson, P. 1970. Information and consumer behavior. Journal of Political Economy 78(2) 311-29.

Obermiller, C. 1988. When do consumers infer quality from price? M. J. Houston, ed., NA - Advances in Consumer Research Volume 15. Association for Consumer Research, 304-310.

Özer, Ö., Y. Zheng. 2012. Behavioral issues in pricing management. Ö. Özer, R. Phillips, eds., The Oxford Handbook of Pricing Management. Oxford University Press, Oxford, 415-465.

Roberts, J.H., J. M. Lattin. 1991. Development and testing of a model of consideration set composition. Journal of Marketing Research 429-440.

Roesler, A-K. 2015. Is ignorance bliss? rational inattention and optimal pricing. Tech. rep.

Sahin, O., R. Wang. 2014. The impact of consumer search cost on assortment planning and pricing. Working Paper. Available at SSRN 2503490.

Shannon, C. E. 1948. The mathematics theory of communication. Bell Syst. Tech. J 27 379-423.

Simon, H. A. 1955. A behavioral model of rational choice. The Quarterly Journal of Economics 69(1) 99-118.

Sims, C. A. 1998. Stickiness. Carnegie-Rochester Conference Series on Public Policy 49(1) 317-356.

Sims, C. A. 2003. Implications of rational inattention. Journal of Monetary Economics 50(3) 665-690.

Sims, C. A. 2006. Rational inattention: Beyond the linear-quadratic case. The American Economic Review 96(2) 158-163.

Song, J. S., Z. Xue. 2007. Demand management and inventory control for substitutable products. Working paper.

Stafford, J. E., B. M. Enis. 1969. The price-quality relationship: An extension. Journal of Marketing Research 6(4) $456-458$.

Stigler, G. J. 1961. The economics of information. Journal of Political Economy 69(3) 213-225.

Stiving, M. 2000. Price-endings when prices signal quality. Management Science 46(12) 1617-1629.

Stock, A., S. Balachander. 2005. The making of a "hot product": A signaling explanation of marketers' scarcity strategy. Management Science 51(8) 1181-1192.

$\mathrm{Su}, \mathrm{X}$. 2008. Bounded rationality in newsvendor models. Manufacturing $\&$ Service Operations Management 10(4) $566-589$. 
Tirole, J. 1988. The theory of industrial organization. MIT press.

Tutino, A. 2011. 'Rational Inattention' Guides Overloaded Brains, Helps Economists Understand Market Behavior. Economic Letter 6(mar) 1-4.

Tutino, A. 2013. Rationally inattentive consumption choices. Review of Economic Dynamics 16(3) 421-439.

Weitzman, M. L. 1979. Optimal search for the best alternative. Econometrica 47(3) 641-654.

Wheatley, J. J., J. S.Y. Chiu. 1977. The effects of price, store image, and product and respondent characteristics on perceptions of quality. Journal of Marketing Research 14(2) 181-186.

Wiederholt, M. 2010. Rational inattention. Steven N. Durlauf, Lawrence E. Blume, eds., The New Palgrave Dictionary of Economics. Palgrave Macmillan, Basingstoke.

Woodford, M. 2009. Information-constrained state-dependent pricing. Journal of Monetary Economics 56(S) $100-124$.

\section{Appendix. Technical Proofs}

Lemma 1 (i) $\bar{p} \geq \alpha q_{H}+(1-\alpha) q_{L}$, and $(i i) \underline{p} \leq \alpha q_{H}+(1-\alpha) q_{L}$.

Proof. We can re-write the inequality in (i) $\ln \left[\alpha e^{q_{H} / \lambda}+(1-\alpha) e^{q_{L} / \lambda}\right] \geq \alpha\left(q_{H} / \lambda\right)+(1-\alpha)\left(q_{L} / \lambda\right)$ substituting for $\bar{p}$. Taking exponents of both sides, we obtain $\alpha e^{q_{H} / \lambda}+(1-\alpha) e^{q_{L} / \lambda} \geq e^{\alpha\left(q_{H} / \lambda\right)+(1-\alpha)\left(q_{L} / \lambda\right)}$, which is true due to the convexity of the function $f(x)=e^{x}$. Similarly, we can simplify the inequality in (ii) as $\ln \left[(1-\alpha) e^{q_{H} / \lambda}+\alpha e^{q_{L} / \lambda}\right] \geq(1-\alpha)\left(q_{H} / \lambda\right)+\alpha\left(q_{L} / \lambda\right)$ after substituting for $\underline{p}$. This inequality holds true based on our argument for part (i).

Lemma $2 x>\frac{1}{\operatorname{coth} x}$ for $x>0$.

Proof. When $x>0$, both $x$ and $\frac{1}{\operatorname{coth} x}$ are increasing in $x\left(\frac{\partial}{\partial x} \frac{1}{\operatorname{coth} x}=(\operatorname{sech} x)^{2} \geq 0\right)$. We also know that $\frac{1}{\operatorname{coth} x}=0$ if $x=0$. Further, note that the rate of increase of $x$ is larger than that of $\frac{1}{\operatorname{coth} x}$, i.e., $(\operatorname{sech} x)^{2} \leq 1$ for all $x>0$. Hence, the inequality given in the lemma is true.

Proof of Proposition 1. First, note that $\dot{\pi}_{0}(p)$ is non-increasing in $p$ since

$$
\frac{\partial \dot{\pi}_{0}(p)}{\partial p}=-\frac{\alpha\left[\operatorname{csch}\left(\frac{p-q_{L}}{2 \lambda}\right)\right]^{2}+(1-\alpha)\left[\operatorname{csch}\left(\frac{p-q_{H}}{2 \lambda}\right)\right]^{2}}{4 \lambda} \leq 0 .
$$

When $p \leq \underline{p}$, we have $\dot{\pi}_{0}(p) \geq 1$. Subsequently $\pi_{0}(p)=1$ and $\pi_{L}(p)=\pi_{H}(p)=1$. Since $\mathcal{R}_{i}(p)=p \times \pi_{i}(p)$ increases in $p$ in this interval, we have $p_{i}^{*} \geq \underline{p}$. Moreover, $\underline{p}$ is increasing in $\alpha$ and hence approaches $q_{L}$ as $\alpha \downarrow 0$, i.e., $\underline{p}>q_{L}$. On the other hand, when $p \geq \bar{p}$, we have $\dot{\pi}_{0}(p) \leq 0$. Hence $\pi_{0}(p)=0$ and also $\pi_{L}(p)=\pi_{H}(p)=0$. Since $\mathcal{R}_{i}(p)=0$ in this interval, $p^{*} \leq \bar{p}$. Furthermore, $\bar{p}$ is increasing in $\alpha$ and approaches $q_{H}$ as $\alpha \uparrow 1$, i.e., $\bar{p}<q_{H}$. 
Proof of Theorem 1. (i) Letting $\xi\left(\pi_{i}\right)=\frac{\pi_{0}}{1-\pi_{0}} \times \frac{1-\pi_{i}}{\pi_{i}}$, we re-write the firm's revenue function as $\mathcal{R}_{i}\left(\pi_{i}\right)=$ $q \pi_{i}+\lambda \pi_{i} \cdot \ln \left[\xi\left(\pi_{i}\right)\right]$. Taking derivatives, we obtain $\frac{\partial^{2} \mathcal{R}_{i}\left(\pi_{i}\right)}{\partial \pi_{i}^{2}}$ as

$$
\frac{\partial^{2} \mathcal{R}_{i}\left(\pi_{i}\right)}{\partial \pi_{i}^{2}}=\frac{2 \lambda \xi^{(1)}\left(\pi_{i}\right)}{\xi\left(\pi_{i}\right)}+\lambda \pi_{i} \times\left\{-\left(\frac{\xi^{(1)}\left(\pi_{i}\right)}{\xi\left(\pi_{i}\right)}\right)^{2}+\frac{\xi^{(2)}\left(\pi_{i}\right)}{\xi\left(\pi_{i}\right)}\right\}
$$

where $\xi^{(1)}(\cdot)$ and $\xi^{(2)}(\cdot)$ denote the first and second derivatives of $\xi\left(\pi_{i}\right)$ with respect to $\pi_{i}$, respectively. From (24), $\mathcal{R}_{i}\left(\pi_{i}\right)$ is concave in $\pi_{i}$ if: (1) $\xi\left(\pi_{i}\right)$ is non-negative, i.e., $\xi\left(\pi_{i}\right) \geq 0,(2) \xi\left(\pi_{i}\right)$ is non-increasing in $\pi_{i}$, i.e., $\xi^{(1)}\left(\pi_{i}\right) \leq 0$, and $(3) \xi\left(\pi_{i}\right)$ is concave in $\pi_{i}$, i.e., $\xi^{(2)}\left(\pi_{i}\right) \leq 0$.

Clearly, $\xi\left(\pi_{i}\right)$ is always non-negative. We next verify that $\xi^{(1)}\left(\pi_{i}\right) \leq 0$ and $\xi^{(2)}\left(\pi_{i}\right) \leq 0$ for $i \in\{H, L\}$. Note that the conditional purchase probabilities $\pi_{H}$ and $\pi_{L}$, using (7), are given by

$$
\pi_{H}=\frac{e^{\left(q_{H}-p\right) / \lambda}}{e^{\left(q_{H}-p\right) / \lambda}+\frac{\left(1-\pi_{0}\right)}{\pi_{0}}} \quad \text { and } \quad \pi_{L}=\frac{e^{\left(q_{L}-p\right) / \lambda}}{e^{\left(q_{L}-p\right) / \lambda}+\frac{\left(1-\pi_{0}\right)}{\pi_{0}}}
$$

respectively. Then, we express $\pi_{L}$ in terms of $\pi_{H}$ as $\pi_{L}=\frac{\pi_{H} e^{q_{L} / \lambda}}{e^{q_{H} / \lambda}-\pi_{H}\left(e^{q_{H} / \lambda}-e^{q_{L} / \lambda}\right)}$, plug into (8) to obtain

$$
\pi_{0}=\alpha \pi_{H}+\frac{(1-\alpha) \pi_{H} e^{q_{L} / \lambda}}{e^{q_{H} / \lambda}-\pi_{H}\left(e^{q_{H} / \lambda}-e^{q_{L} / \lambda}\right)}
$$

and consequently $\xi\left(\pi_{H}\right)$ as follows

$$
\xi\left(\pi_{H}\right)=\frac{\alpha\left(1-\pi_{H}\right) e^{q_{H} / \lambda}+\left(1-\alpha\left(1-\pi_{H}\right)\right) e^{q_{L} / \lambda}}{\left(1-\alpha \pi_{H}\right) e^{q_{H} / \lambda}+\alpha \pi_{H} e^{q_{L} / \lambda}}
$$

As a result, $\xi^{(1)}\left(\pi_{H}\right)$ and $\xi^{(2)}\left(\pi_{H}\right)$ are calculated as

$$
\xi^{(1)}\left(\pi_{H}\right)=-\frac{\alpha(1-\alpha)\left(e^{q_{H} / \lambda}-e^{q_{L} / \lambda}\right)^{2}}{\left(\left(1-\alpha \pi_{H}\right) e^{q_{H} / \lambda}+\alpha \pi_{H} e^{q_{L} / \lambda}\right)^{2}} \quad \text { and } \quad \xi^{(2)}\left(\pi_{H}\right)=-\frac{2 \alpha^{2}(1-\alpha)\left(e^{q_{H} / \lambda}-e^{q_{L} / \lambda}\right)^{3}}{\left(\left(1-\alpha \pi_{H}\right) e^{q_{H} / \lambda}+\alpha \pi_{H} e^{q_{L} / \lambda}\right)^{3}}
$$

respectively. We observe that $\xi^{(1)}\left(\pi_{H}\right) \leq 0$ and $\xi^{(2)}\left(\pi_{H}\right) \leq 0$, since $0<\alpha<1,0 \leq \pi_{H} \leq 1$ and $q_{H}>q_{L}$.

Similarly, we express $\pi_{H}$ in terms of $\pi_{L}$ as $\pi_{H}=\frac{\pi_{L} e^{q_{H} / \lambda}}{e^{q_{L} / \lambda}+\pi_{L}\left(e^{q_{H} / \lambda}-e^{q_{L} / \lambda}\right)}$, plug into (8) to get

$$
\pi_{0}=\frac{\alpha \pi_{L} e^{q_{H} / \lambda}}{e^{q_{L} / \lambda}+\pi_{L}\left(e^{q_{H} / \lambda}-e^{q_{L} / \lambda}\right)}+(1-\alpha) \pi_{L}
$$

Then $\xi\left(\pi_{L}\right)$ is calculated as

$$
\xi\left(\pi_{L}\right)=\frac{\left(\pi_{L}+\alpha\left(1-\pi_{L}\right)\right) e^{q_{H} / \lambda}+(1-\alpha)\left(1-\pi_{L}\right) e^{q_{L} / \lambda}}{(1-\alpha) \pi_{L} e^{q_{H} / \lambda}+\left(1-(1-\alpha) \pi_{L}\right) e^{q_{L} / \lambda}}
$$

Accordingly, $\xi^{(1)}\left(\pi_{L}\right)$ and $\xi^{(2)}\left(\pi_{L}\right)$ follow as

$\xi^{(1)}\left(\pi_{L}\right)=-\frac{\alpha(1-\alpha)\left(e^{q_{H} / \lambda}-e^{q_{L} / \lambda}\right)^{2}}{\left((1-\alpha) \pi_{L} e^{q_{H} / \lambda}+\left(1-(1-\alpha) \pi_{L}\right) e^{q_{L} / \lambda}\right)^{2}}$ and $\xi^{(2)}\left(\pi_{L}\right)=-\frac{2 \alpha(1-\alpha)^{2}\left(e^{q_{H} / \lambda}-e^{q_{L} / \lambda}\right)^{3}}{\left((1-\alpha) \pi_{L} e^{q_{H} / \lambda}+\left(1-(1-\alpha) \pi_{L}\right) e^{q_{L} / \lambda}\right)^{3}}$

respectively. Once again, we find that $\xi^{(1)}\left(\pi_{L}\right) \leq 0$ and $\xi^{(2)}\left(\pi_{L}\right) \leq 0$, as $0<\alpha<1,0 \leq \pi_{L} \leq 1$ and $q_{H}>q_{L}$.

As a result, we conclude that $\xi^{(1)}\left(\pi_{i}\right) \leq 0$ and $\xi^{(2)}\left(\pi_{i}\right) \leq 0$ for $i \in\{H, L\}$. Hence, $\mathcal{R}_{i}\left(\pi_{i}\right)$ is concave in $\pi_{i}$.

(ii) First, suppose that product quality is high. Since $\pi_{H}(p)$ is given by

$$
\pi_{H}(p)=\frac{\pi_{0}(p) e^{\left(q_{H}-p\right) / \lambda}}{\pi_{0}(p) e^{\left(q_{H}-p\right) / \lambda}+\left(1-\pi_{0}(p)\right)},
$$

using (12), we can express $\pi_{H}(p)$ in closed-form for $\underline{p} \leq p \leq \bar{p}$ as follows:

$$
\pi_{H}(p)=\frac{e^{q_{H} / \lambda}}{\alpha}\left[\frac{1}{e^{q_{H} / \lambda}-e^{q_{L} / \lambda}}-\frac{1-\alpha}{e^{q_{H} / \lambda}-e^{p / \lambda}}\right]
$$


Therefore, $\mathcal{R}_{H}(p)=p \times \pi_{H}(p)=\frac{p e^{q_{H} / \lambda}}{\alpha}\left[\frac{1}{e^{q_{H} / \lambda}-e^{q_{L} / \lambda}}-\frac{1-\alpha}{e^{q} H^{\prime \lambda}-e^{p / \lambda}}\right]$. The second derivative of $\mathcal{R}_{H}(p)$ w.r.t. $p$ is

$$
\frac{\partial^{2} \mathcal{R}_{H}(p)}{\partial p^{2}}=-\frac{(1-\alpha)\left[\operatorname{csch}\left(\frac{p-q_{H}}{2 \lambda}\right)\right]^{2}}{4 \lambda^{2} \alpha} \times\left[2 \lambda-p \cdot \operatorname{coth}\left(\frac{p-q_{H}}{2 \lambda}\right)\right] .
$$

Since $-\frac{(1-\alpha)\left[\operatorname{csch}\left(\frac{p-q_{H}}{2 \lambda}\right)\right]^{2}}{4 \lambda^{2} \alpha} \leq 0$ for all $0<\alpha<1, \lambda>0$, and $\operatorname{coth}\left(\frac{p-q_{H}}{2 \lambda}\right) \leq 0$ for $p \leq q_{H}$, we have $\frac{\partial^{2} \mathcal{R}_{H}(p)}{\partial p^{2}} \leq 0$. As a result, $\mathcal{R}_{H}(p)$ is concave in $p$ over $\underline{p} \leq p \leq \bar{p}$.

Next, suppose that product quality is low. As we have

$$
\pi_{L}(p)=\frac{\pi_{0}(p) e^{\left(q_{L}-p\right) / \lambda}}{\pi_{0}(p) e^{\left(q_{L}-p\right) / \lambda}+\left(1-\pi_{0}(p)\right)},
$$

we can express $\pi_{L}(p)$ in closed-form for $p \leq p \leq \bar{p}$ as follows:

$$
\pi_{L}(p)=\frac{e^{q_{L} / \lambda}}{1-\alpha}\left[\frac{\alpha}{e^{p / \lambda}-e^{q_{L} / \lambda}}-\frac{1}{e^{q_{H} / \lambda}-e^{q_{L} / \lambda}}\right]
$$

Hence, $\mathcal{R}_{L}(p)=p \times \pi_{L}(p)=\frac{p e^{q_{L} / \lambda}}{1-\alpha}\left[\frac{\alpha}{e^{p / \lambda}-e^{q_{L} / \lambda}}-\frac{1}{e^{q_{H} / \lambda}-e^{q_{L} / \lambda}}\right]$. The first derivative of $\mathcal{R}_{L}(p)$ w.r.t. $p$ is

$$
\frac{\partial \mathcal{R}_{L}(p)}{\partial p}=-\frac{e^{q_{L} / \lambda}}{1-\lambda}\left[\frac{1}{e^{q_{H} / \lambda}-e^{q_{L} / \lambda}}+\frac{e^{p / \lambda} \alpha(p-\lambda)+e^{q_{L} / \lambda} \alpha \lambda}{\left(e^{p / \lambda}-e^{q_{L} / \lambda}\right)^{2} \lambda}\right]
$$

Clearly, $e^{p / \lambda} \alpha(p-\lambda)+e^{q_{L} / \lambda} \alpha \lambda>0$ if $p \geq \lambda$. Now assume $p<\lambda$. In order to have $e^{p / \lambda} \alpha(p-\lambda)+e^{q_{L} / \lambda} \alpha \lambda \geq 0$, we need show that $\lambda e^{q_{L} / \lambda} \geq(\lambda-p) e^{p / \lambda}$ which implies $\frac{\lambda}{\lambda-p} \geq e^{\left(p-q_{L}\right) / \lambda}$. As $p \downarrow q_{L}$ (note $q_{L}<p<\lambda$ ), the latter inequality is satisfied (strictly). Also observe that LHS and RHS increase in $p$, but there exists no real $p$ that solves the said inequality as an equality. Since both LHS and RHS are continuous, the inequality is always satisfied, hence $e^{p / \lambda} \alpha(p-\lambda)+e^{q_{L} / \lambda} \alpha \lambda>0$. As a result, for all $p \in[\underline{p}, \bar{p}]$, we have $\frac{\partial \mathcal{R}_{L}(p)}{\partial p}<0$, and $\mathcal{R}_{L}(p)$ is decreasing in $p$. To check for convexity, we calculate second derivative of $\mathcal{R}_{L}(p)$ w.r.t. $p$ as

$$
\frac{\partial^{2} \mathcal{R}_{L}(p)}{\partial p^{2}}=-\frac{(1-\alpha)\left[\operatorname{csch}\left(\frac{p-q_{L}}{2 \lambda}\right)\right]^{2}}{4 \lambda^{2} \alpha} \times\left[2 \lambda-p \cdot \operatorname{coth}\left(\frac{p-q_{L}}{2 \lambda}\right)\right] .
$$

First note that $-\frac{(1-\alpha)\left[\operatorname{csch}\left(\frac{p-q_{L}}{2 \lambda}\right)\right]^{2}}{4 \lambda^{2} \alpha} \leq 0$ for all $0<\alpha<1$ and $\lambda>0$. Moreover, based on Lemma 2 , we have $\operatorname{coth}\left(\frac{p-q_{L}}{2 \lambda}\right)>\frac{2 \lambda}{p-q_{L}}$ (note that $\left.p-q_{L}>0\right)$. If we re-write this inequality, we obtain $2 \lambda-\left(p-q_{L}\right) \operatorname{coth}\left(\frac{p-q_{L}}{2 \lambda}\right) \leq 0$. Hence, we also have $2 \lambda-p \operatorname{coth}\left(\frac{p-q_{L}}{2 \lambda}\right) \leq 0$ for $p>q_{L}$. As a result, $\frac{\partial^{2} \mathcal{R}_{L}(p)}{\partial p^{2}} \geq 0$ and $\mathcal{R}_{L}(p)$ is convex in $p$.

Proof of Corollary 1. Theorem 1 and Proposition 1 together directly imply the result.

Proof of Proposition 2. We first show that $p_{L}^{*}\left(=\mathcal{R}_{L}^{*}\right)$ is increasing in $\lambda$. We know that $p_{L}^{*}=q_{L}+q_{H}-$ $\lambda \ln \left[(1-\alpha) e^{q_{H} / \lambda}+\alpha e^{q_{L} / \lambda}\right]$. Taking the derivative of $p_{L}^{*}$ w.r.t. $\lambda$ we check if

$$
\frac{\partial p_{L}^{*}}{\partial \lambda}=\frac{(1-\alpha) q_{H} e^{q_{H} / \lambda}+\alpha q_{L} e^{q_{L} / \lambda}}{\lambda\left[(1-\alpha) e^{q_{H} / \lambda}+\alpha e^{q_{L} / \lambda}\right]}-\ln \left[(1-\alpha) e^{q_{H} / \lambda}+\alpha e^{q_{L} / \lambda}\right] \geq 0 .
$$

We re-write the above condition as follows:

$$
(1-\alpha) \frac{q_{H}}{\lambda} e^{q_{H} / \lambda}+\alpha \frac{q_{L}}{\lambda} e^{q_{L} / \lambda} \geq\left[(1-\alpha) e^{q_{H} / \lambda}+\alpha e^{q_{L} / \lambda}\right] \ln \left[(1-\alpha) e^{q_{H} / \lambda}+\alpha e^{q_{L} / \lambda}\right]
$$

Note that the RHS of the condition is of the form $x \ln (x)$ which is increasing convex for $x>0$. Hence, the above condition directly follows from the definition of convexity.

In order to prove the result regarding the high-quality product, first recall that as $\lim _{\lambda \downarrow 0} \mathcal{R}_{H}^{*}=q_{H}>\alpha q_{H}+(1-$ a) $q_{L}=\lim _{\lambda \uparrow \infty} \mathcal{R}_{H}^{*}$. To show that optimal profit is non-monotone in this region, we will establish the existence 
Author: Pricing When Customers Have Limited Attention

of a finite $\lambda_{c}$ such that $\mathcal{R}_{H}^{*}$ is increasing in $\lambda$ on $\lambda \geq \lambda_{c}$. This will follow when we prove that on $\lambda \geq \lambda_{c}$ for some finite $\lambda_{c}$, it is optimal for the firm serve the entire market $\left(\pi_{H}^{*}=1\right)$, implying that the optimal price and revenue will be the same as the low-quality product (which is increasing in $\lambda$ as proved above). We will utilize the concavity of $\mathcal{R}\left(\pi_{H}\right)$ shown in Theorem 1 for this result. Specifically, we are going to investigate the first derivative of $\mathcal{R}\left(\pi_{H}\right)$ w.r.t. $\pi_{H}$ as $\pi_{H} \uparrow 1$ and show that it is increasing in $\lambda$ and changes sign once (at $\lambda_{c}$ ) from negative to positive. From concavity, this implies that $\pi_{H}^{*}=1$ for $\lambda \geq \lambda_{c}$.

Observe that the first of derivative of $\mathcal{R}\left(\pi_{H}\right)$ w.r.t. $\pi_{H}$ is given as

$$
q_{H}+\lambda \ln \left[\xi\left(\pi_{H}\right)\right]+\lambda \pi_{H} \frac{\xi^{(1)}\left(\pi_{H}\right)}{\xi\left(\pi_{H}\right)},
$$

which in the limit as $\pi_{H} \uparrow 1$ becomes (after some manipulation)

$$
q_{H}+\left\{\lambda \ln \left[\frac{e^{q_{L} / \lambda}}{(1-\alpha) e^{q_{H} / \lambda}+\alpha e^{q_{L} / \lambda}}\right]-\lambda \alpha(1-\alpha) \frac{\left(e^{q_{H} / \lambda}-e^{q_{L} / \lambda}\right)^{2}}{e^{q_{L} / \lambda}\left((1-\alpha) e^{q_{H} / \lambda}+\alpha e^{q_{L} / \lambda}\right)}\right\}
$$

Let's denote the terms within the bracket \{\} as $B$. Since $\frac{e^{q_{L} / \lambda}}{(1-\alpha) e^{q_{H} / \lambda}+\alpha e^{q_{L} / \lambda}} \leq 1$ and $\frac{\left(e^{q_{H} / \lambda}-e^{q_{L} / \lambda}\right)^{2}}{e^{q_{L} / \lambda}\left((1-\alpha) e^{q_{H} / \lambda}+\alpha e^{q_{L} / \lambda}\right)} \geq 0$, we have $B \leq 0$. Moreover, $B$ is also increasing in $\lambda$. To show this, we examine the first derivative of $B$ with respect to $\lambda$ which is given as

$$
\begin{aligned}
\frac{\partial B}{\partial \lambda}= & \underbrace{\frac{(1-\alpha)\left(q_{H}-q_{L}\right) e^{q_{H} / \lambda}}{\lambda\left((1-\alpha) e^{q_{H} / \lambda}+\alpha e^{q_{L} / \lambda}\right)}+\ln \left[\frac{e^{q_{L} / \lambda}}{(1-\alpha) e^{q_{H} / \lambda}+\alpha e^{q_{L} / \lambda}}\right]}_{C} \\
& +\underbrace{\frac{e^{-q_{L} / \lambda}\left(e^{q_{H} / \lambda}-e^{q_{L} / \lambda}\right)(1-\alpha) \alpha}{\lambda\left((1-\alpha) e^{q_{H} / \lambda}+\alpha e^{q_{L} / \lambda}\right)^{2}}}_{D} \\
& \underbrace{\times\left((1-\alpha)\left(q_{H}-q_{L}-\lambda\right) e^{2 q_{H} / \lambda}+\alpha \lambda e^{2 q_{L} / \lambda}+\left(\left(q_{H}-q_{L}\right)(1+\alpha)+\lambda(1-2 \alpha)\right) e^{\left(q_{H}+q_{L}\right) / \lambda}\right)}_{E}
\end{aligned}
$$

Firstly, $C$ is decreasing in $\lambda$ since

$$
\frac{\partial C}{\partial \lambda}=-\frac{\alpha(1-\alpha)\left(q_{H}-q_{L}\right)^{2} e^{\left(q_{H}+q_{L}\right) / \lambda}}{\lambda^{3}\left((1-\alpha) e^{q_{H} / \lambda}+\alpha e^{q_{L} / \lambda}\right)^{2}} \leq 0
$$

and $\lim _{\lambda \rightarrow \infty} C=0$. Hence, $C \geq 0$ for all $\lambda \geq 0$. Secondly, $D \geq 0$. Thirdly, $E$ is convex in $q_{H}$ for any given $\lambda, \alpha$ and $q_{L}$ since

$$
\frac{\partial^{2} E}{\partial q_{H}^{2}}=\frac{e^{q_{H} / \lambda}\left(4 e^{q_{H} / \lambda}\left(q_{H}-q_{L}\right)(1-\alpha)+e^{q_{L} / \lambda}\left(\left(q_{H}-q_{L}\right)(1+\alpha)+3 \lambda\right)\right)}{\lambda^{2}} \geq 0
$$

$E$ is minimized at $q_{H}=q_{L}$, assuming the value 0 . Hence, $E \geq 0$.

Having established that $B$ is increasing in $\lambda$, we further note that $\lim _{\lambda \rightarrow 0} B=-\infty$ and $\lim _{\lambda \rightarrow \infty} B=-(1-\alpha)\left(q_{H}-q_{L}\right)$. This means that in the limit as $\pi_{H} \uparrow 1, \partial R\left(\pi_{H}\right) / \partial \pi_{H}=q_{H}+B$ is increasing in $\lambda$, approaches $-\infty$ as $\lambda \downarrow 0$ and approaches $q_{L}+\alpha\left(q_{H}-q_{L}\right)>0$ when $\lambda \uparrow \infty$. Hence, there exists a finite threshold $\lambda=\lambda_{c}$ above which $\pi_{H}^{*}=1$.

Proof of Proposition 3. Based on Corollary 1, we know that $\mathcal{R}_{L}^{*}$ (and $p_{L}^{*}$ ) is given by $q_{L}+q_{H}-\lambda \ln [(1-$ $\left.\alpha) e^{q_{H} / \lambda}+\alpha e^{q_{L} / \lambda}\right]$. Since

$$
\frac{\partial \mathcal{R}_{L}^{*}}{\partial \alpha}=\frac{\lambda\left(e^{q_{H} / \lambda}-e^{q_{L} / \lambda}\right)}{(1-\alpha) e^{q_{H} / \lambda}+\alpha e^{q_{L} / \lambda}} \geq 0
$$

and

$$
\frac{\partial \mathcal{R}_{L}^{*}}{\partial q_{L}}=\frac{(1-\alpha) e^{q_{H} / \lambda}}{(1-\alpha) e^{q_{H} / \lambda}+\alpha e^{q_{L} / \lambda}} \geq 0,
$$


$\mathcal{R}_{L}^{*}$ (and $p_{L}^{*}$ ) is increasing in both $\alpha$ and $q_{L}$.

We use the envelope theorem to show the impact of $\alpha$ and $q_{L}$ on $\mathcal{R}_{H}^{*}$ (since its closed-form solution does not exist). Using our derivations for the proof of Theorem 1 , we have $\mathcal{R}_{H}(p)=p \times \pi_{H}(p)=$ $\frac{p e^{q_{H} / \lambda}}{\alpha}\left[\frac{1}{e^{q_{H} / \lambda}-e^{q_{L} / \lambda}}-\frac{1-\alpha}{e^{q_{H} / \lambda}-e^{p / \lambda}}\right]$. Then

$$
\frac{\partial \mathcal{R}_{H}(p)}{\partial \alpha}=\frac{p e^{q_{H} / \lambda}\left(e^{p / \lambda}-e^{q_{L} / \lambda}\right)}{\left(e^{q_{H} / \lambda}-e^{p / \lambda}\right)\left(e^{q_{H} / \lambda}-e^{q_{L} / \lambda}\right) \alpha^{2}} \geq 0 \quad \text { for } \underline{p} \leq p \leq \bar{p}
$$

and

$$
\frac{\partial \mathcal{R}_{H}(p)}{\partial q_{L}}=\frac{e^{\left(q_{H}+q_{L}\right) / \lambda} p}{\left(e^{q_{H} / \lambda}-e^{q_{L} / \lambda}\right)^{2} \alpha^{2}} \geq 0 \quad \text { for } \underline{p} \leq p \leq \bar{p}
$$

On the other hand, $\left.\frac{\partial p}{\partial \alpha}\right|_{p=p_{H}^{*}}$ and $\left.\frac{\partial p}{\partial q_{L}}\right|_{p=p_{H}^{*}}$ can be obtained through the implicit function theorem. First note that

$\frac{\partial \mathcal{R}_{H}(p)}{\partial p \partial \alpha}=\frac{e^{q_{H} / \lambda}}{\left(e^{q_{H} / \lambda}-e^{p / \lambda}\right)^{2}\left(e^{q_{H} / \lambda}-e^{q_{L} / \lambda}\right) \lambda \alpha^{2}}\left(\lambda e^{p}\left(e^{q_{H} / \lambda}+e^{q_{L} / \lambda}-e^{p / \lambda}\right)+p e^{p / \lambda}\left(e^{q_{H} / \lambda}-e^{q_{L} / \lambda}\right)\right) \geq 0 \quad$ for $\underline{p} \leq p \leq \bar{p}$

and

$$
\frac{\partial \mathcal{R}_{H}(p)}{\partial p \partial q_{L}}=\frac{p e^{\left(q_{H}+q_{L}\right) / \lambda}}{\alpha \lambda\left(e^{q_{H} / \lambda}-e^{q_{L} / \lambda}\right)^{2}} \geq 0
$$

We also know $\frac{\partial^{2} \mathcal{R}_{H}(p)}{\partial p^{2}} \leq 0$ since $\mathcal{R}_{H}(p)$ is concave in $p$ based on Theorem 1. As a result, $-\frac{\partial \mathcal{R}_{H}(p)}{\partial p \partial \alpha} / \frac{\partial^{2} \mathcal{R}_{H}(p)}{\partial p^{2}} \geq 0$ and $-\frac{\partial \mathcal{R}_{H}(p)}{\partial p \partial q_{L}} / \frac{\partial^{2} \mathcal{R}_{H}(p)}{\partial p^{2}} \geq 0$.

Proof of Theorem 2. Note that for any separating equilibrium, there exists $\hat{p}_{L}, \hat{p}_{H} \in\left[q_{L}, q_{H}\right]$ and $\hat{p}_{H} \neq \hat{p}_{L}$ such that $\hat{\sigma}_{H}\left(\hat{p}_{H}\right)>0, \hat{\sigma}_{L}\left(\hat{p}_{H}\right)=0, \hat{\sigma}_{H}\left(\hat{p}_{L}\right)=0$, and $\hat{\sigma}_{L}\left(\hat{p}_{L}\right)>0$. Bayesian beliefs together with $(5)$ imply $\hat{\mu}\left(\hat{p}_{H}\right)=1$ and $\hat{\mu}\left(\hat{p}_{L}\right)=0$. Let $\hat{\mathcal{R}}_{H} \doteq \mathcal{R}_{H}\left(\hat{p}_{H}, \hat{\mu}\left(\hat{p}_{H}\right)\right)$ and $\hat{\mathcal{R}}_{L} \doteq \mathcal{R}_{L}\left(\hat{p}_{L}, \hat{\mu}\left(\hat{p}_{L}\right)\right)$. For such prices to be a separating equilibrium, firm optimality conditions have to hold. Together with the fact that the consumer will not process information if $\mu \in\{0,1\}$ :

$$
\begin{aligned}
& \hat{\mathcal{R}}_{H}=\mathcal{R}_{H}\left(\hat{p}_{H}, 1\right) \geq \mathcal{R}_{H}\left(\hat{p}_{L}, 0\right)=\mathcal{R}_{L}\left(\hat{p}_{L}, 0\right)=\hat{\mathcal{R}}_{L}, \\
& \hat{\mathcal{R}}_{L}=\mathcal{R}_{L}\left(\hat{p}_{L}, 0\right) \geq \mathcal{R}_{L}\left(\hat{p}_{L}, 1\right)=\mathcal{R}_{H}\left(\hat{p}_{L}, 1\right)=\hat{\mathcal{R}}_{H} .
\end{aligned}
$$

Putting these together, we need to have

$$
\hat{\mathcal{R}}_{H}=\mathcal{R}_{H}\left(\hat{p}_{H}, 1\right)=\hat{p}_{H} 1_{\hat{p}_{H} \leq q_{H}}=\hat{\mathcal{R}}_{L}=\mathcal{R}_{L}\left(\hat{p}_{L}, 0\right)=\hat{p}_{L} 1_{\hat{p}_{L} \leq q_{L}},
$$

which clearly cannot happen.

In contrast, a pooling equilibrium is always supported by the belief structure $\mu(p)=\alpha$ if $p=\hat{p}$ and $\mu(p)=0$ otherwise. Using our previous results, it can be established that such a price (range) exists. To see this, first note that $\mathcal{R}_{H}(p, 0)=\mathcal{R}_{L}(p, 0) \leq q_{L}$. Hence, for both type firms the maximum profit that can be earned when a non-equilibrium price used is $q_{L}$. Now, from Theorem 1 we know that $\mathcal{R}_{L}(p, \alpha)$ is decreasing and convex on $p \in[\underline{p}, \bar{p}]$. Furthermore $\mathcal{R}_{L}(\underline{p}, \alpha)=\underline{p}>q_{L}$ (Proposition 1) and $\mathcal{R}_{L}(\bar{p}, \alpha)=0$, so there exists a unique $p=\tilde{p}(\alpha)=$ $\left\{\max p: \mathcal{R}_{L}(p, \alpha) \geq q_{L}\right\}$. Now consider any price $\hat{p} \in[\underline{p}, \tilde{p}(\alpha)], \mathcal{R}_{L}(\hat{p}, \alpha) \geq \mathcal{R}_{L}(p, 0)$. Since $\mathcal{R}_{H}(p, \alpha)>\mathcal{R}_{L}(p, \alpha)$ always, we also have that $\mathcal{R}_{H}(\hat{p}, \alpha) \geq \mathcal{R}_{H}(p, 0)$. As a result, firm optimality is achieved and both types of firms would prefer to use $\hat{p}$ over any other price. Correspondingly, $\mu(\hat{p})=\alpha$ and $\mu(p)=0$ for any other price.

Proof of Proposition 4. To see part $(i)$, suppose that a pooling equilibrium price $\hat{p}$ exists. From Theorem 1 , we know that $\mathcal{R}_{L}(p, \alpha)$ is decreasing convex. Hence if $\underline{p}<\hat{p}$, then there exists a price $p^{\prime}$ such that $\mathcal{R}_{L}\left(p^{\prime}, \alpha\right)>$ 
Author: Pricing When Customers Have Limited Attention

$\mathcal{R}_{L}(\hat{p}, \alpha)$. Then by assumption it must be that $\mu\left(p^{\prime}\right) \geq \alpha$. Nonetheless, we have $\mathcal{R}_{L}\left(p^{\prime}, \mu\left(p^{\prime}\right)\right)>\mathcal{R}_{L}(p, \alpha)$ violating firm optimality. So now consider the case $\hat{p}=\underline{p}$. In this case firm optimality is not violated for the low-quality firm. However, when $\lambda<\lambda_{c}$ we know from Proposition 2 that $\pi_{H}^{*}<1$, implying that the maximizer of $\mathcal{R}_{H}(p, \alpha)$ is greater than $\underline{p}$. Given the concavity of $\mathcal{R}_{H}(p, \alpha)$, there is a $p^{\prime}>\underline{p}$ such that $\mathcal{R}_{H}\left(p^{\prime}, \alpha\right)>\mathcal{R}_{H}(p, \alpha)$, which from the refinement assumption would imply $\mu\left(p^{\prime}\right) \geq \alpha$. This time we have $\mathcal{R}_{H}\left(p^{\prime}, \mu\left(p^{\prime}\right)\right)>\mathcal{R}_{H}(p, \alpha)$ violating firm optimality for the high-quality firm. Hence, a pooling equilibrium cannot exist for $\lambda<\lambda_{c}$. For part (ii), note from Proposition 2 that when $\lambda \geq \lambda_{c}$, we have $\pi_{H}^{*}=\pi_{L}^{*}=1$, meaning $\underline{p}=\arg \max _{p} \mathcal{R}_{H}(p, \alpha)=\arg \max _{p} \mathcal{R}_{L}(p, \alpha)$. Hence, for any $\hat{p}>\underline{p}$, we can apply the same logic above to show that firm optimality would be violated under the refinement. Hence, the only price surviving the refinement is $\hat{p}=p$.

Proof of Proposition 5. Attentive customers purchase the product with quality $q$ and price $p$ with probability 1 as long as $p \leq q$. First, suppose that $q=q_{H}$. If the firm chooses to serve both attentive and inattentive customers, based on Proposition 1, it would have to set price $p$ such that $p \leq \lambda\left[\alpha e^{q_{H} / \lambda}+(1-\alpha) e^{q_{L} / \lambda}\right]<q_{H}$. Otherwise, inattentive customers will choose the outside option without processing any information. In this case, the firm earns $\beta p \pi_{H}(p)+(1-\beta) p$. Let $\tilde{p}_{H}$ denote the price which maximizes this particular revenue function. Based on Theorem 1, $\tilde{p}_{H}$ is uniquely defined. If the firm chooses to serve attentive customers only, it would set the price at $q_{H}$ and earn $(1-\beta) q_{H}$. Accordingly, the firm's optimal strategy is to serve both segments only if the proportion of attentive customers in the market is higher than a certain threshold, i.e., $\beta \geq \frac{q_{H}-\tilde{p}_{H}}{q_{H}-\left(1-\pi\left(\tilde{p}_{H}\right)\right) \tilde{p}_{H}}$. If this is the case, the firm sets the price as $\tilde{p}_{H}$. Otherwise, it is optimal for the firm to serve attentive customers only and set the price as $q_{H}$.

Next assume $q=q_{L}$. If the firm chooses to serve both attentive and inattentive customers, it would have to set the product price as $q_{L}$, since attentive customers would not buy the product at a higher price. At this particular price, we know that all inattentive customers would also purchase the product, i.e., $\pi\left(q_{L}\right)=1$; hence, the firm earns $q_{L}$. If the firm chooses to serve inattentive customers only, it would set the price as $p_{L}^{*}=q_{H}+q_{L}-\lambda\left[(1-\alpha) e^{q_{H} / \lambda}+\alpha e^{q_{L} / \lambda}\right]$ and earn $\beta\left(q_{H}+q_{L}-\lambda\left[(1-\alpha) e^{q_{H} / \lambda}+\alpha e^{q_{L} / \lambda}\right]\right)$, as shown in Corollary 1. As a result, the optimal strategy of the firm is to serve both customer segments by setting the price as $q_{L}$ only if the proportion of inattentive customers is below a certain threshold, i.e., $\beta \leq \frac{q_{L}}{\left(q_{L}+q_{H}-\lambda \ln \left[(1-\alpha) e^{q_{H} / \lambda}+\alpha e^{q_{L} / \lambda}\right]\right)}$. Otherwise, the firm should only sell to inattentive customers and set the price as $p_{L}^{*}$.

Proof of Proposition 6. First we define $\underline{p}_{k}$ and $\bar{p}_{k}$ as the lower and upper bounds on the optimal price that maximizes the firm's revenue from customers with belief $\alpha_{k}, k \in\{1,2\}$, as described in Proposition 1 . If $\alpha_{2} \geq \frac{\alpha_{1} e^{q_{L} / \lambda}}{\left(1-\alpha_{1}\right)^{q_{H} / \lambda}+\alpha_{1} e^{q_{L} / \lambda}}$ ), we have $\bar{p}_{1} \geq \bar{p}_{2} \geq \underline{p}_{1} \geq \underline{p}_{2}$; otherwise $\bar{p}_{1} \geq \underline{p}_{1}>\bar{p}_{2} \geq \underline{p}_{2}$. For brevity, we assume that the first condition is satisfied (a similar proof can easily be extended to the second case). Then, we express the firm's revenue function as follows

$$
\mathcal{R}_{i}(p, \alpha)=\left\{\begin{array}{lll}
p & \text { if } p<\underline{p} \\
\gamma p+(1-\gamma) p \pi_{i, 2}(p) & \text { if } \underline{p}_{2} \leq p<\underline{p}_{1} \\
\gamma p \pi_{i, 1}(p)+(1-\gamma) p \pi_{i, 2}(p) & \text { if } \underline{p}_{1} \leq p<\bar{p}_{2} \\
\gamma p \pi_{i, 1}(p) & \text { if } \bar{p}_{2} \leq p \leq \bar{p}_{1} \\
0 & \text { if } p>\bar{p}_{1}
\end{array} \quad \text { for } i \in\{H, L\}\right.
$$

Since $\pi_{H, 1}(p)$ and $\pi_{H, 2}(p)$ are concave in $p$ based on Theorem $1, \mathcal{R}_{H}(p, \alpha)$ is piecewise concave in $p$, and a unique solution to $p_{H}^{*}$ exists. On the other hand, $\mathcal{R}_{L}(p, \alpha)$ is increasing if $p<\underline{p}_{2}$, convex if $\underline{p}_{2} \leq p<\underline{p}_{1}$, and convex decreasing if $\underline{p}_{1} \leq p<\bar{p}_{1}$. Hence, $p_{L}^{*} \in\left\{\underline{p}_{1}, \underline{p}_{2}\right\}$. Following from (25), the seller would set $p_{L}^{*}=\underline{p}_{2}$ if $\underline{p}_{2}>\gamma \underline{p}_{1}+(1-\gamma) \underline{p}_{1} \pi_{L, 2}\left(\underline{p}_{1}\right)$, or equivalently if $\gamma<\underline{\gamma}=\frac{\underline{p}_{2}-\underline{p}_{1} \pi_{L, 2}\left(\underline{p}_{1}\right)}{\underline{p}_{1}\left(1-\pi_{L, 2}\left(\underline{p}_{1}\right)\right.}$. If $\gamma \geq \underline{\gamma}$, then $p_{L}^{*}=\underline{p}_{1}$. 


\section{Recent ESMT Working Papers}

ESMT No.

Taking 'some' of the mimicry out of the adoption process: Quality management and strategic substitution

16-05

Joseph A. Clougherty, University of Illinois at Urbana-Champaign, CEPR-London Michat Grajek, ESMT European School of Management and Technology

Oz Shy

Consumer choice under limited attention when options have different information costs

Frank Huettner, ESMT European School of Management and Technology Tamer Boyacı, ESMT European School of Management and Technology

Yalçın Akçay, College of Administrative Sciences and Economics, Koç University

Are level $\mathbf{3}$ fair values reflected in firm value? Evidence from European banks

Katja Kisseleva, ESMT European School of Management and Technology

Daniela Lorenz, Free University, Berlin

Design for reusability and product reuse under radical innovation

Tamer Boyac1, ESMT European School of Management and Technology

Vedat Verter, Desautels Faculty of Management, McGill University

Michael R. Galbreth, Moore School of Business, University of South Carolina

Pricing when customers have limited attention

Tamer Boyacı, ESMT European School of Management and Technology

Yalçın Akçay, College of Administrative Sciences and Economics, Koç University

Regional state aid control in Europe: A legal and economic assessment

Hans W. Friederiszick, ESMT European School of Management and Technology Massimo Merola, Bonelli Erede, College of Europe of Bruges

Fund flows inducing mispricing of risk in competitive financial markets

Axel Stahmer, ESMT European School of Management and Technology 